Running Head: INTELLIGENCE, CREATIVITY, AND THE BRAIN

Intelligence and Creativity Share a Common Cognitive and Neural Basis

\author{
Emily Frith ${ }^{1}$, Daniel B. Elbich ${ }^{2}$, Alexander P. Christensen ${ }^{3}$, Monica D. Rosenberg ${ }^{4}$,
}

Qunlin Chen ${ }^{2,5}$, Michael J. Kane ${ }^{3}$, Paul J. Silvia ${ }^{3}$, Paul Seli ${ }^{6}, \&$ Roger E. Beaty ${ }^{2}$

${ }^{1}$ Department of Psychology, University of Mississippi, USA

${ }^{2}$ Department of Psychology, Pennsylvania State University, USA

${ }^{3}$ Department of Psychology, University of North Carolina at Greensboro, USA

${ }^{4}$ Department of Psychology, University of Chicago, IL, USA

${ }^{5}$ School of Psychology, Southwest University, China

${ }^{6}$ Department of Psychology and Neuroscience, Duke University, USA

Word Count: 18,482

Author Note

R.E.B. is supported by a grant from the National Science Foundation [DRL-1920653]. This research was supported by grant RFP-15-12 to R.E.B, M.J.K, and P.J.S. from the Imagination Institute (www.imagination-institute.org), funded by the John Templeton Foundation. The opinions expressed in this publication are those of the authors and do not necessarily reflect the view of the Imagination Institute or the John Templeton Foundation.

Correspondence should be addressed to Roger Beaty, Department of Psychology, 140 Moore Building, University Park, PA, 16801, USA; rebeaty@psu.edu. 


\begin{abstract}
Are intelligence and creativity distinct abilities, or do they rely on the same cognitive and neural systems? We sought to quantify the extent to which intelligence and creative cognition overlap in brain and behavior by combining machine learning of fMRI data and latent variable modeling of cognitive ability data in a sample of young adults $(N=186)$ who completed a battery of intelligence and creative thinking tasks. The study had three analytic goals: (a) to assess contributions of specific facets of intelligence (e.g., fluid and crystallized intelligence) and general intelligence to creative ability (i.e., divergent thinking originality), $(b)$ to model whole-brain functional connectivity networks that predict intelligence facets and creative ability, and (c) to quantify the degree to which these predictive networks overlap in the brain. Using structural equation modeling, we found moderate to large correlations between intelligence facets and creative ability, as well as a large correlation between general intelligence and creative ability $(r=.63)$. Using connectomebased predictive modeling, we found that functional brain networks that predict intelligence facets overlap to varying degrees with a network that predicts creative ability, particularly within the prefrontal cortex of the executive control network. Notably, a network that predicted general intelligence shared $46 \%$ of its functional connections with a network that predicted creative ability-including connections linking executive control and salience/ventral attention networks-suggesting that intelligence and creative thinking rely on similar neural and cognitive systems.
\end{abstract}

Keywords: creativity, connectome-based predictive modeling, divergent thinking, intelligence, structural equation modeling 


\section{Intelligence and Creativity Share a Common Cognitive and Neural Basis}

Intelligence is a mental faculty of undeniable importance. To successfully reason, people must draw from previous experiences to adapt to novel circumstances. In 1937, Bingham argued that the meaning of intelligence should be interpreted as the ability to "solve new problems." Other theorists suggest that intelligence permits imagination, abstraction from rote experiences, and manipulation of ambiguity to make sense of the world (Feurestein et al., 1979, 2002; Guilford, 1967; Jensen, 1998; Terman, 1922) -all of which are cognitive processes that have been historically associated with creative ability (Abraham, 2018). The apparent overlap between intelligence and creative cognition has motivated decades of psychometric research aiming to characterize this relationship, with more recent evidence pointing to a considerable overlap between these two cognitive abilities (Silvia, 2015).

Several questions remain, however, about the nature of the intelligence-creativity relationship, including whether intelligence influences creative thinking through general or specific abilities (e.g., visuospatial reasoning, verbal fluency) and whether intelligence and creative thinking rely on a similar neural architecture in the brain. In the present research, we aimed to address these questions by combining structural equation modeling of multiple intelligence facets with machine learning of functional brain data obtained during creative task performance. This approach allowed us to quantify the extent to which creative cognition and intelligence overlap in brain and behavior.

\section{Intelligence and Creative Cognition}

Broadly, creative thinking encompasses the ability to generate novel ideas and solutions that are task- and context-appropriate and effective (Diedrich et al., 2015; Runco \& Jaegar, 2012). According to the controlled-attention theory of creative cognition, goal-directed idea generation is governed by top-down control of mental processes that promote the strategic search for task-relevant responses (Beaty \& Silvia, 2012, 2013; Benedek, Franz, Heene, \& Neubauer, 2012; Jauk, Benedek, \& Neubauer, 2014; Silvia \& Beaty, 2012). The quality of creative ideas is thought to depend largely upon individual differences in executive function, a collection of cognitive processes involved in strategic control over thought and action. In contrast, the associative theory of creative cognition posits that novel ideas emerge from automatic, 
combinatory processes that are contingent upon underlying semantic knowledge structures, and that individual differences in creative thinking ability reflect variation in the organization of, and access to, concepts within semantic networks (Mednick, 1962; Kenett \& Faust, 2019). More recently, arguments for a dual-process perspective were raised to account for the complementary interaction between executive and associative processes in creative cognition. According to this integrated framework, controlled executive abilities are needed to access associative elements and further inhibit (in the case of salient but unoriginal elements), adapt (transform old experiences into new ideas), and combine (link disparate concepts into novel responses) knowledge into high-quality ideas (Beaty et al., 2014b; Benedek \& Neubauer, 2013).

One way to measure creative cognition is with divergent thinking assessments, which require people to generate original ideas based on an open-ended prompt, such as generating uncommon uses for common objects (Guilford, 1967; Silvia, Martin, \& Nusbaum, 2009). Laboratory-based creativity research often uses divergent thinking tasks as indications of broader creative potential (Runco, \& Acar, 2012), as they moderately predict the frequency of real-world creative behaviors (Jauk, Benedek, \& Neubauer, 2014; Beaty et al., 2013). Unlike convergent thinking tasks, which are evaluated in terms of speed and accuracy (Cropley, 2006), divergent thinking tasks encourage a variety of novel responses to stimuli that are inherently unexpected and tend to vary across individuals (Dygert \& Jarosz, 2019). In this context, divergent thinking can be viewed as a cognitive ability that is supported both by associative and executive processes, which work together to activate diffuse semantic knowledge and override salient (but unoriginal) mental representations to guide the generation of novel and task-appropriate solutions (Beaty et al., 2014; Silvia, Nusbaum, \& Beaty, 2017).

Notably, however, performance on laboratory-assessed divergent thinking tasks does not always predict creative accomplishment (Barron \& Harrington, 1981; Cropley, 2000; Plucker, 1999; Runco \& Acar, 2012; Sternberg \& Lubart, 1996; Zeng, Proctor, \& Salvendy, 2011). One reason for this lack of consensus between measurement and real-world achievement is that creativity — as assessed with common verbal tasks of divergent thinking - is not exclusively domain-general. For example, a musician may use divergent thinking primarily in an auditory domain, meaning that a divergent thinking assessment in the 
verbal domain may not adequately capture her potential for domain-specific musical creativity (Barron \& Harrington, 1981). Taken together, although domain-general divergent thinking measures serve as viable indices of creative potential, such assessments do not comprehensively capture creativity as a singular entity because these measures alone cannot account for the multitude of factors that influence broader creative abilities and accomplishments (Silvia, Winterstein, \& Willse, 2008), including personality, motivation, social context, domain experience, and intelligence (Barron \& Harrington, 1981; Sternberg \& Lubart, 1991).

Intelligence is a hierarchical construct reflecting multiple, correlated general abilities that hinge on executing goal-directed behavior (Gottfredson, 1997; Jensen, 1998). Mental operations, including reasoning, planning, problem-solving, and environmental adaptation, interact to reflect broader concepts of intelligence (Goldstein et al., 2015; Gottfredson, 1997; Neisser et al., 1996). Rather than merely questioning whether intelligence and creative cognition are associated, then, modern scientific inquiry is increasingly focused on delineating mechanisms to explain the nature of this often-observed relationship (Plucker et al., 2015; Plucker \& Renzulli, 1999; Silvia, 2015). Although a wealth of research has investigated relationships between intelligence and creative thinking, a lack of empirical resolution remains, perhaps because creativity and intelligence are complex constructs that have been subjected to a wide range of conceptualizations. However, despite considerable variability in the operationalization of these constructs, modern research efforts continue to focus on clarifying the creativity-intelligence relationship by identifying cognitive and neural operations that may play a role in intelligent and creative behavior (Jung \& Vartanian, 2018; Sternberg \& Kaufman, 2011).

Sternberg and O'Hara (1999) proposed three interpretations of the intelligence-creativity relation: (a) creative thinking is an element of human intelligence, such that various cognitive factors including divergent thinking, memory, and complex reasoning are integral to intelligent thinking (Guilford, 1967); (b) intelligence is an element of creative thinking, meaning that higher-order cognitions, such as cognitive flexibility, inhibition, and goal-directed problem-solving, as well as the capacity for knowledge acquisition, comprehension, and retention, play essential roles in directing creative cognition (Sternberg, 1996), or (c) 
creative thinking and intelligence share overlapping features. The most widely endorsed perspective is that creative thinking and intelligence overlap; that is, creative thinking is similar to standard problem-solving, but is not identical (Kim, 2005). Similarities between creativity and intelligence include the ability to solve difficult problems by adopting novel perspectives, and inhibiting obvious, yet inappropriate responses. However, unlike intelligence assessments, the creative process often lacks a clear end-point or systematic set of rules which may be followed to achieve an optimal solution. Another key difference lies in quantifying intelligent and creative cognitions. Intelligence is often evaluated on the basis of "rightness" or correctness, whereas creativity is evaluated on the basis of "goodness" or the quality of original thoughts and behavior (Shouksmith, 1973; Sternberg \& O’Hara, 1999). Importantly, both intelligent and creative thoughts can be right and original, which reinforces the dynamic integration of these constructs (Sternberg, 1999).

Although controversy exists in understanding and defining intelligence (see Carroll, 1993; Kovacs \& Conway, 2016; Shipstead, Harrison, \& Engle, 2016; Spearman, 1904; Sternberg, 1984), the widely influential Carroll-Horn-Carroll taxonomic model of intelligence (CHC; McGrew, 2005) proposes that intelligence is comprised of correlated abilities within a hierarchical framework. These abilities span a continuum capturing general (e.g., higher-order $g$ ) intelligence, with distinctions among broad (e.g., fluid reasoning) and specific (e.g., speed of reasoning) factors, which are both separable from and subsumed into g. A major strength of the CHC model is its flexibility in accounting for a range of intermediate abilities and domain-specific knowledge and skills that define human cognition (McGrew, 2005, 2009). A weakness of the $\mathrm{CHC}$ model, as a descriptive taxonomy, is that it neither illuminates whether $g$ is a unitary cognitive or biological process, nor identifies the mechanisms that causally link $g$ to lower-order cognitive abilities (Conway \& Kovacs, 2015). However, recent network-based models of intelligence suggest that higherorder $g$ does not cause variation in lower-order factors, but rather that individual differences in intelligence reflect an emergent property of many cognitive processes that overlap or interact in solving any particular intellectual problem (Schmank, Goring, Kovacs, \& Conway, 2019). In this vein, a summation of lowerorder factors gives rise to a psychometric index of intelligence rather than a general intelligence causing 
variation in the lower-order factors (Van Der Maas et al., 2006). These "mutualism" perspectives, suggesting that $g$ does not represent a unitary psychological construct (Conway \& Kovacs, 2015; Kovacs \& Conway, 2016; Shipstead, Harrison, \& Engle, 2016; Van Der Maas et al., 2006), may support the argument that widespread observations of creativity-intelligence relationships in the literature reflect multiple processes working together to influence the extent to which creativity and intelligence overlap at the cognitive level.

Another feature of contemporary research on creative cognition and intelligence has been an emphasis on distinguishing general from specific cognitive abilities, with general intelligence constituting higher-order $g$ and specific abilities reflecting $g$ 's intelligence's lower-order facets (McGrew, 2005). One lower-order facet of intelligence relevant to creative thinking is broad retrieval ability (Gr), which reflects the ability to strategically retrieve relevant concepts from long-term memory (e.g., verbal fluency tasks requiring people to recall all the animals they can in 2 min; Benedek et al., 2014a). Past work has found consistent effects of Gr on divergent thinking performance (Benedek et al., 2012, b; Lee \& Therriault, 2013; Silvia, Beaty \& Nusbaum, 2013), suggesting that divergent thinking involves controlled retrieval and the selection of information from memory. Additionally, crystallized intelligence (Gc) - the ability to employ acquired knowledge (e.g., vocabulary) to solve problems (Cattell, 1963)-correlates with divergent thinking performance (Cho et al., 2010), suggesting that creative thinking benefits from access to more learned information. Furthermore, complex reasoning and higher-order control processes reflecting fluid intelligence (Gf; Cattell, 1963) — the ability to solve novel problems with reasoning —also are associated with divergent-thinking originality (Benedek et al., 2012; Silvia, 2008; Silvia \& Beaty, 2012) and adoption of creative strategies in divergent thinking tasks (Nusbaum \& Silvia, 2011). In contrast, the relationship between creative thinking and the ability to mentally manipulate and reorganize three-dimensional objects, or visuospatial intelligence (Gv; Schneider \& McGrew, 2012), is less explored; however, research has shown that visuospatial ability may contribute to individual differences in real-world creative achievements (i.e., patents and publications; Kell et al., 2013), as well as success in scientific, technological, engineering, and mathematical domains (Wai et al., 2009). Taken together, evidence indicates that specific facets of 
intelligence may support performance on creative thinking tasks, pointing to an overlap between these cognitive abilities at the level of behavior.

\section{Brain Systems Supporting Creative Cognition and Intelligence}

The neural basis of creative cognition and intelligence has been examined using multiple neuroimaging methods, from structural MRI (Jung et al., 2010) to diffusion tensor imaging (DTI; Kenett et al., 2018; Takeuchi et al., 2010, 2020) to functional MRI (fMRI; Benedek et al., 2018; Jung, 2014). Recent fMRI investigations of creative cognition have implicated functional connectivity between large-scale brain networks, particularly the default network (midline and lateral parietal cortices) and the executive control network (lateral prefrontal and anterior inferior parietal cortices). On the one hand, the default network primarily supports internally-focused attention and self-referential cognition, such as episodic retrieval, mental simulation, and spontaneous thought (Buckner \& DiNicola, 2019). On the other hand, the executive network primarily supports externally focused attention and executively demanding cognition, such as working memory, inhibitory control, and task-set shifting (Niendam et al., 2012). A third network—the salience or ventral attention network-plays an important role in switching between the default and executive control networks (Uddin, 2015).

Although the default and executive networks support seemingly opposing modes of attention and cognition, default-executive cooperation is among the most consistent findings in creativity neuroscience (for reviews, see Beaty et al., 2016, 2019), with studies reporting functional connectivity between default and executive networks during creative task performance-including divergent thinking (Adnan et al., 2019; Vartanian et al., 2018), musical improvisation (Pinho et al., 2014), and poetry composition (Liu et al., 2015) - and individual differences analyses reporting positive correlations between creative ability and default-executive connectivity (Beaty et al., 2014a, 2018; Takeuchi et al., 2017). Notably, despite the consistency of this connectivity profile in the literature, to date the cognitive mechanisms that modulate brain connectivity during creative task performance remain largely uncharacterized.

A wealth of behavioral evidence has demonstrated links between intelligence and creative cognition (see Benedek et al., 2014b; Plucker et al., 2015; Silvia, 2008, 2015; Sternberg \& O’Hara, 1999). 
Indeed, specific facets of intelligence may account for individual differences in functional connectivity during divergent thinking. For example, substantial evidence has linked Gf to the executive network via the "parietal-frontal" theory of intelligence (i.e., P-FIT; Jung \& Haier, 2007), supporting the hypothesis that a core function of the executive network is goal-directed behavior, and that this function is expressed by individual differences in fluid intelligence (Barbey et al., 2013). Initial evidence obtained by van den Heuval et al. (2009) suggests that fluid intelligence relates to efficiency of information processing at the level of whole-brain organization, consistent with seminal theories of intelligence and neural efficiency (Haier et al., 1988; Neubauer \& Fink, 2009). Network neuroscience research has since extended this work by demonstrating a more complex role of the executive network-in coordination with other functional brain networks - to support goal-directed behavior (Barbey, 2018). According to a recent network neuroscience theory of intelligence proposed by Barbey (2018), intelligence supports goal-directed behavior by coordinating whole-brain network dynamics, not by modulating the activation of singular brain regions or networks.

Functional imaging research has investigated how intelligence relates to individual differences in brain function during goal-directed task performance. Early fMRI activation studies found that Gf modulates task-relevant brain regions, particularly during executively demanding tasks requiring working memory and attentional control (Gray et al., 2003; Lee et al., 2006). More recently, network neuroscience approaches have examined how intelligence interacts with brain reconfiguration during task performance. Schultz and Cole (2016) found that higher Gf was linked to less task-induced brain network configuration across several cognitive tasks (compared to baseline resting-state organization), extending early univariate activation findings on intelligence and neural efficiency (Neubauer \& Fink, 2009) to the level of brain network dynamics. By combining multiple measures of intelligence with task fMRI data from the Human Connectome Project, Sripada et al. (2019) found that higher-order $g$ strongly predicted default and executive network activity during a demanding working memory task. Specifically, as the task increased in difficulty, intelligence was related to greater separation of default and executive networks, such that more intelligent participants adapted to the task demands by forming distinct modules of default and executive 
regions. These findings suggest that intelligence may modulate whole-brain dynamics during executivelydemanding cognitive tasks.

Individual differences in intelligence and creative cognition have also been studied using functional connectivity-based prediction methods (e.g., connectome-based predictive modeling, CPM; Shen et al., 2017; Rosenberg et al., 2017). Such data-driven methods can reliably predict behavioral phenotypes including personality (Hsu et al., 2018), attentional control (Rosenberg et al., 2018), fluid intelligence (Finn et al., 2015), and creative cognition (Beaty et al., 2018) from functional connectivity patterns. In a recent CPM study of creative cognition (i.e., divergent thinking), Beaty et al. (2018) found that participants with higher scores on creativity assessments showed stronger functional connections between frontal and parietal regions within executive, salience, and default networks. Regarding intelligence, Finn et al. (2015) and others (e.g., Jiang et al., 2020) have used connectome prediction methods to reliably predict individual fluid intelligence using resting-state fMRI data, demonstrating that variation in intelligence can be reliably detected from patterns of whole-brain connectivity. Finn and colleagues found that although the frontoparietal/executive control network emerged as a distinctive feature predictive of individual intelligence, the predictive patterns were complex and distributed across the whole brain. Taken together, increasing evidence indicates that similar neural systems may support intelligence and creative cognition, but the extent to which these cognitive abilities overlap in the brain remains unclear.

\section{The Present Research}

Intelligence appears to play an important role in creative cognition, including lower-order facets of intelligence (e.g., Gf) contributing to divergent thinking performance (Avitia \& Kaufman, 2014; Benedek et al., 2014; Forthmann et al., 2019; Jauk et al., 2013; Karwowski et al., 2016; Silvia et al., 2013). Related neuroimaging research has highlighted common and distinct neural systems supporting intelligence and divergent thinking, particularly within frontal and parietal regions of the executive control network (Barbey, 2018; Chen et al., 2016; Finn et al., 2015; Jung \& Chohan, 2019; Kenett et al., 2018). These findings are consistent with contemporary theories of creative cognition that emphasize executive and strategic cognitive processes involved in the controlled retrieval and combination of stored representations (Beaty et 
al., 2016; Benedek et al., 2014a; Chen et al., 2016; Volle, 2018). However, an open question concerns how creative cognition relates to both lower-order, more specific facets (i.e., Gf, Gr, Gc, Gv) and high-order general intelligence (i.e., g). Moreover, to our knowledge, no neuroimaging study has directly examined how intelligence relates to brain dynamics during creative thinking. Understanding whether intelligence modulates brain processes relevant to creative cognition could yield new insights into the relationship between intelligence and creativity.

The present research sought to clarify the intelligence-creativity relation by combining latent variable modeling of behavioral data with functional MRI data acquired during performance on a divergent creative thinking task (using the participant sample described in Beaty et al. 2018). We assessed multiple lower-order facets of intelligence with theoretical relevance to creative cognition-fluid intelligence, crystalized intelligence, broad retrieval ability, and visuospatial intelligence - and leveraged structural equation models to model relationships among these cognitive abilities and divergent thinking. Notably, a majority of past research has assessed lower-order intelligence facets on creative task performance, and fewer studies have examined effects of higher-order $g$ beyond zero-order correlations and composite averages, which tend to attenuate effect sizes and underestimate statistical relationships between constructs (McNeish \& Wolf, 2019; Silvia et al., 2008). Our study thus provides a first look at how creative cognition relates to both specific facets of intelligence and higher-order $g$, providing a comprehensive analysis of the intelligence-creativity relationship.

Another major goal of the current study was to examine how intelligence and creative cognition overlap in the brain. To this end, we analyzed fMRI data acquired during divergent thinking, and we used a data-driven prediction method (i.e., CPM) to identify functional brain connections that predict performance on both intelligence and creative thinking tasks. Specifically, we reanalyzed neuroimaging data from Beaty et al. (2018), which used CPM to predict individual creative thinking performance across multiple datasets. We provide a novel extension of CPM that aims to identify functional connections that relate to both intelligence and creativity by assessing common and distinct network predictions of both cognitive abilities. Moreover, prior predictive models of intelligence such as CPM have only assessed $g$ 
and fluid intelligence; here, we examine higher-order and lower-order facts of $\mathrm{g}$ with a novel application (i.e., fMRI data collected during a creative thinking task).

Consistent with recent behavioral evidence (e.g., Benedek et al., 2014; Silvia et al., 2013), we hypothesized that specific intelligence facets would correlate positively with divergent thinking ability. Moreover, we expected to find a large latent correlation between $g$ and divergent thinking, given previous studies reporting effects of individual lower-order facets (e.g., Beaty et al., 2014; Forthmann et al., 2019; Jauk et al., 2013; Silvia et al., 2013). Regarding neural effects, we hypothesized that the strength of functional connectivity between executive, salience, and default regions would predict divergent thinking ability, consistent with our prior analyses of these fMRI data (Beaty et al., 2018); however, we aimed to replicate and extend our recent CPM study using a new functional atlas (Schaefer et al., 2018) to determine whether prediction of divergent thinking is impervious to parcellation choice, given recent machine learning evidence demonstrating that prediction accuracy (and corresponding functional connections) are sensitive to the choice of brain atlas (Dadi et al., 2019; Schaefer et al., 2018). Critically, we hypothesized that intelligence and creativity (i.e., divergent thinking) would share common predictive brain features within frontal brain regions of the executive control network.

\section{Materials and Methods}

\section{Participants}

This study was approved by the institutional review board at the University of North Carolina at Greensboro (UNCG). Participants (total $N=186,129$ women, mean age $=22.74$ years, $S D=6.37$ ) were recruited as part of a larger, multi-visit study designed to evaluate individual differences in creativity (see Beaty et al., 2018). They provided written informed consent prior to data collection and were compensated up to $\$ 100$ based on their level of study completion. Participants were right-handed with normal or corrected-to-normal vision, and they were not enrolled in the study if they reported a history of neurological disorder, cognitive disability, or medication and other drugs known to affect the central nervous system. A subset of participants was excluded from the neuroimaging analysis due to excessive head movement (mean framewise displacement $>.5 \mathrm{~mm}, n=4$; Power et al., 2012), hardware or software issues associated with 
fMRI data collection (e.g., E-Prime crash), and incomplete behavioral data (e.g., completing lab assessments but not the fMRI task). After exclusions, the final sample for fMRI analysis was 171 (119 females, mean age $=22.73$ years, $S D=6.22$ ), which differed slightly from Beaty and colleagues' (2018) final sample size of 163 , due to the availability of additional cases. The behavioral analyses include data from the full sample of participants $(n=186)$.

\section{Intelligence Assessment}

Participants completed a series of behavioral measures during two laboratory visits. The first visit involved neuroimaging and a subset of the behavioral measures; the second visit (approximately 1-week later) involved completing the remaining behavioral measures. All measures were administered using the MediaLab software package.

Crystallized intelligence $(\boldsymbol{G c})$. Participants completed two measures of vocabulary knowledge, which are indicative of Gc (Kan et al., 2011): (a) the advanced vocabulary test (18 items), and (b) the extended range vocabulary test (24 items; ETS Kit of Factor-Referenced Cognitive Tests; Ekstrom et al., 1976). Participants were given a total of seven minutes to complete both tasks, which required selecting the synonym of a word target from a list of possible answers. Each task was scored for the total number of correctly solved problems.

Fluid intelligence (Gf). Participants completed three measures of Gf: (a) the number series task, (b) the letter sets task, and (c) the matrices task. The number series task (Thurstone, 1938) required identifying a pattern that dictates a series of presented numbers by selecting the next number in the sequence (15 items, 5 minutes); the task was scored for the sum of correctly reported numbers. The letter sets task (Ekstrom et al., 1976) required identifying a set of four letters that violate a rule dictating the larger set (16 items, 4 minutes); the task was scored for the sum of correctly identified rule violations. The series completion task from the Culture Fair Intelligence Test (CFIT; Cattell \& Cattell 1961/2008) presented a series of three changing progressive images, and participants were asked to select a fourth image that most appropriately completed the series of images (13 items, 3 minutes); the task was scored for the sum of correct images chosen. 
Broad retrieval ability $(\boldsymbol{G r})$. Participants completed five verbal fluency tasks that assess two components of broad retrieval ability: ideational fluency and associational fluency (Carroll, 1993; Silvia et al., 2013). Ideational fluency required the generation of category exemplars for the categories (a) animal, (b) fruit/vegetable, and (c) occupation (Ardila et al., 2006). Participants were given one minute for each task and were asked to "write down [type] as many animals [fruits/vegetables, or occupations] as you can." Responses consisting of non-category members were excluded, and root variations were converged (e.g., dogs to $d o g$ ) from the final analysis using the SemNetCleaner package (Christensen \& Kenett, 2019) in $\mathrm{R}$ (R Core Team, 2019). Responses were analyzed categorically, with "1" corresponding to a valid response and " 0 " corresponding to no response. Associational fluency tasks required participants to generate synonyms for the target terms hot and good. Participants were instructed to "write down [type] as many synonyms for hot (good) as you can" in one-minute. Procedures were identical to those employed for the category tasks, with non-synonym responses excluded from the final analysis, and binary scores recorded for each valid response " 1 " and no response " 0. .

Visuospatial intelligence (Gv). Participants completed three measures of Gv that assess the ability to mentally manipulate visual stimuli. For the (a) paper-folding task (Ekstrom et al., 1976), participants were presented with a series of individual pictorial stimuli. Each trial depicts a square piece of paper that is folded one or more times, with a hole punched into it. This assessment required participants to determine where the holes would be located if the paper were unfolded, given five potential alternatives (10 items, 3 minutes); this task was scored for the total number of correct alternatives selected. The (b) block rotations task also measured abstract spatial ability (Berger, Gupta, Berger, \& Skinner, 1990). Participants were shown a rotated, three-dimensional target block image. Participants were then asked to select one of five three-dimensional block choices that matched the shape of the target block, despite being presented at a different angle of rotation (10 items, 8 minutes); this task was scored for the total number of correct selections. For the (c) cube comparisons task (Ekstrom et al., 1976; Thurstone, 1938), participants were asked to report whether two three-dimensional cubes were equivalent or different, across various spatial 
orientations (42 items, 6 minutes); this task was scored for the total number of correct comparisons (see Supplemental Table 1 for descriptive statistics of the intelligence tasks).

\section{fMRI Task Procedures and Divergent Thinking Assessment}

To assess creative cognition, we combined behavioral and event-related fMRI measurement of divergent thinking (see Beaty et al., 2018). During functional imaging, participants completed several trials of the Alternative Uses Task (AUT; Guilford, 1967); they also completed a semantic control condition that was not analyzed here (see Beaty et al., 2018). The AUT measures divergent creative thinking, or the ability to generate novel responses after being presented with a single, open-ended prompt to generate creative uses for common objects (e.g., a brick). For the present experiment, participants completed 23 AUT trials, requiring one alternative use for each of the 23 trials. The trial structure included: (a) a jittered fixation cross (4-6 s), (b) a condition cue (3 s), (c) a silent response generation phase (12 s), and (d) a response production phase requiring participants to speak their response into an MRI-compatible microphone (5 s; cf., Benedek et al., 2014a, 2018; Beaty et al., 2018). Responses were logged by an experimenter for subsequent analysis of creative thinking quality. Prior to the fMRI experiment, an experimenter provided instructions for both tasks and participants were given several practice items. Participants were asked to use the response generation phase to imagine creative uses for the given object and then verbally supply their most creative idea during the response generation phase (Beaty et al., 2018). Specifically, participants were instructed to "be creative" and "to come up with something clever, humorous, original, compelling, or interesting (Beaty et al., 2014b).”

Following the fMRI experiment, as part of the larger project, participants completed a battery of cognitive and questionnaire measures (including intelligence tasks) over the course of two days. The battery included two AUT trials (with the objects box and rope) which were not included in the pool of object items from the fMRI task. The purpose of the post-scan AUT assessment was to assess divergent thinking performance in a more conventional testing environment, and to compare performance on traditional divergent thinking tasks with fMRI task performance. Here, instead of allotting 12 seconds to produce a single idea (as in the scanner), participants were given 2 minutes to continuously generate creative object 
uses by typing their ideas into a text field via MediaLab. Again, participants were instructed to "be creative" and "to come up with something clever, humorous, original, compelling, or interesting (Beaty et al., 2014b)."

Because we were primarily interested in assessing relations between intelligence and creative cognition, we focused our divergent thinking measurement on the creative quality of ideas. We thus used the widely used subjective scoring approach (Silvia et al., 2008) to assign originality ratings to ideas generated in the scanner and during the post-scan behavioral session. Participants' responses were anonymized and merged into one master file prior to the blinded scoring protocol. Four trained raters provided originality scores for the 23 AUT trials using a Likert-type rating scale of a 1 (not at all creative) to 5 (very creative; Silvia et al., 2008). The same four blinded raters scored the creative quality of behavioral AUT responses for each participant (see Supplemental Table 1 for inter-rater correlations).

\section{Analysis Plan and Model Specification}

The present research had two analytic aims: (a) to model contributions of lower-order/specific and higher-order/general intelligence facets to divergent thinking using latent variable models and (b) using CPM, to identify functional brain connections that predict both intelligence and divergent thinking. Regarding the behavioral aim, we specified and estimated a series of latent variable models using maximum likelihood estimation in Mplus 7.31. Gc, Gf, Gr, and $G v$ latent variables were formed using their respective behavioral tasks as indicators. The factor variances were fixed to 1 , and the loadings for the two Gc indicators were constrained to be equal. Divergent thinking performance in the scanner was modeled as a latent variable with the four subjective ratings (one average score per rater per subject) as indicators. Likewise, laboratory divergent thinking performance in response to the "box" and "rope" prompts were modeled as separate latent variables with the four subjective ratings as indicators (Beaty et al., 2018). All task variables were standardized prior to analysis. The standardized effects are presented in the $r$ metric and can be interpreted using the conventional small (.10), medium (.30), and large (.50) guidelines (Cumming, 2012).

\section{MRI Data Acquisition and Preprocessing}


Participants completed the tasks in a single fMRI run. Whole-brain imaging was performed on a 3T Siemens Magnetom MRI system (Siemens Medical Systems) using a 16-channel head coil. BOLDsensitive $\mathrm{T} 2 *$-weighted functional images were acquired using a single shot gradient-echo echo-planar imaging (EPI) pulse sequence $\left[\right.$ repetition time $(\mathrm{TR})=2,000 \mathrm{~ms}$, echo time $(\mathrm{TE})=30 \mathrm{~ms}$, flip angle $=78^{\circ}$, 32 axial slices, $3.5 \times 3.5 \times 4.0 \mathrm{~mm}$, distance factor $0 \%$, field of view $($ FoV $)=192 \times 192 \mathrm{~mm}$, interleaved slice ordering] and corrected online for head motion. The first two volumes were discarded to allow for T1 equilibration effects. Visual stimuli were presented using E-Prime and viewed through a mirror attached to the head coil. In addition to functional imaging, a high resolution T1 scan was acquired for anatomic normalization. Preprocessing of the anatomical and functional data were performed using fMRIPrep1.4.1rc1 (Esteban, et al., 2019); note that our prior CPM study, on a subset of the data analyzed here (Beaty et al., 2018), did not use fMRIPrep to preprocess MRI data as it was not yet available.

Anatomical data preprocessing. The T1-weighted (T1w) image was skull-stripped and corrected for intensity non-uniformity (INU) using ANTs v.2.2.0 (Avants et al., 2008). Brain tissue segmentation of cerebrospinal fluid (CSF), white-matter (WM), and gray-matter (GM) was performed on the brain-extracted T1w using FAST in FSL v.5.0.9 (Zhang et al., 2001). Brain surfaces were reconstructed using FreeSurfer v.6.0.1 (Dale et al., 1999), and the brain mask estimated previously was refined with a custom variation of Mindboggle (Klein et al., 2017). Volume-based spatial normalization to one standard space (MNI152NLin2009cAsym; Fonov et al., 2009) was performed through nonlinear registration with ANTs, using brain-extracted versions of both $\mathrm{T} 1 \mathrm{w}$ reference and the $\mathrm{T} 1 \mathrm{w}$ template.

Functional data preprocessing. For each BOLD run per subject, first a reference volume and its skull-stripped version were generated using a custom methodology of fMRIPrep. The BOLD reference was then co-registered to the T1w reference FreeSurfer, which implements boundary-based registration (Greve $\&$ Fischl, 2009). Co-registration was configured with nine degrees of freedom to account for distortions remaining in the BOLD reference. Head-motion parameters with respect to the BOLD reference (transformation matrices, and six corresponding rotation and translation parameters) are estimated before any spatiotemporal filtering using FSL (Jenkinson et al., 2002). BOLD runs were slice-time corrected using 
AFNI (Cox \& Hyde, 1997). The BOLD time-series were then resampled into MNI space using ANTs. Framewise displacement (FD) and three region-wise global signals extracted from CSF, the WM, and the whole-brain masks, respectively, were also computed as confound regressors (Satterthwaite et al., 2013; Power et al., 2014).

Functional network construction and preprocessing. Whole-brain functional brain networks were constructed using the Functional Connectivity (CONN) toolbox in MATLAB (Whitfield-Gabrieli \& NietoCastanaon, 2012). To extend our recent study (Beaty et al., 2018) using a different functional brain atlas (i.e., Shen et al., 2017) and a slightly larger sample, we sought to determine whether divergent thinking prediction is agnostic to parcellation by constructing networks using the Schaefer parcellation (Schaefer et al., 2018; note that, unlike the Shen atlas, the Schaeffer atlas does not include the subcortex and cerebellum). To improve comparison with our prior work, we selected a network parcellation with a comparable number of nodes (i.e., 300) with coverage of the seven major canonical intrinsic connectivity networks (i.e., executive control, salience/ventral attention, default, dorsal attention, visual, somatomotor, and limbic). BOLD signal was extracted from each of the 300 regions during the thinking period of the AUT (23 trials, $12 \mathrm{~s}$; collapsing across trials), and bivariate correlations were computed between each pair of ROIs, resulting in a $300 \times 300$ correlation matrix for each participant. In a first-level analysis, white matter and CSF masks, along with first-order derivatives of motion, were entered as confounds and regressed from the ROI timeseries. Additional preprocessing steps included high-pass filtering, linear detrending, and regression of outlying functional volumes (FD > .5; Power et al., 2012). The onsets and durations of the verbal response periods were regressed to account for expected artifacts related to participant vocalization.

Connectome-based predictive modeling. As in our prior work (Beaty et al., 2018), CPM was used to identify functional connectivity networks related to divergent thinking ability. Here, we provide a novel extension of this recent study (and the CPM approach) by identifying functional connectivity networks that predict both lower-order/specific and higher-order/general intelligence facets using fMRI data acquired during the divergent thinking task. This allowed us to quantify the degree of overlap between intelligence 
and creativity in the brain by identifying functional brain connections that predict both constructs (cf., Lake et al., 2019; Rosenberg et al., 2018; Wu et al., 2020).

CPM was executed using the standard pipeline employed in our past work on divergent thinking (Beaty et al., 2018) as well as several other studies (Finn et al., 2015; Rosenberg et al., 2018). A thorough and concise tutorial on the CPM approach was recently published by Shen and colleagues (Shen et al., 2017). CPM builds a model of functional connectivity features to predict behavioral scores in new (unseen) participants using leave-one-out cross validation. Here, the behavioral scores were creativity and intelligence factors extracted from a latent variable model (cf., Beaty et al., 2018) that used the full sample. The CPM pipeline involves: (a) leaving out data from a test participant for cross-validation, (b) correlating a behavioral vector (e.g., creativity or intelligence scores) with all edges in the remaining participants' functional connectivity matrices, (c) thresholding these matrices to retain significantly positively and negatively correlated edges $(p<.01)$, (d) summing these thresholded edges to obtain measures of functional connectivity strength (i.e., positive and negative networks), (e) fitting a linear model (regression) to estimate the brain-behavior relationship for the positive and negative networks, and (f) applying the model to data from the left-out participant to predict their behavioral score. The resulting predictive models are tested for statistical significance by correlating the model-predicted and observed behavior scores with the magnitude of the correlation reflecting the explanatory power of the model. Because the leave-one-out folds are not independent, we conducted permutation tests for all analyses by randomly shuffling the behavior scores (creativity and intelligence facets) 1,000 times and rerunning each CPM, creating a null distribution of $r$ values; the $p$ values of the empirical correlation values (based on their respective null distribution) were computed by dividing the number of permutation $r$ values greater than the empirical $r$ value by 1,000 . All CPM models below report these permutation $p$ values in parentheses alongside corresponding $r$ values.

We estimated separate predictive models for the intelligence and creativity variables using the latent factor scores and divergent thinking functional connectivity matrices. To examine the extent of overlap between the creativity and intelligence networks, we computed the proportion of shared predictive 
features in the final CPM masks. This approach allowed us to directly probe the intelligence-creativity relationship by testing whether specific brain connections similarly predict these cognitive abilities.

\section{Results}

Descriptive statistics for the intelligence factor and divergent thinking measures are displayed in Tables 1 and 2, respectively. Pearson correlations are visualized with a heatmap in Figure 1; a numerical correlation matrix is presented in Supplemental Table 1.

\section{Intelligence and Divergent Thinking Measurement Models}

We first specified a measurement model of the four lower-order intelligence factors: Gf, Gc, Gr, and Gv. This model fit the data well: $\chi^{2}(60 \mathrm{df}) 88.999, p=.009$; CFI .941; RMSEA $=.051$ [90\% CI: .026, $.072] ;$ SRMR $=.058$. Figure 2 depicts the model and inter-correlations between the latent factors. All indicators showed significant loadings on their respective latent variables. Consistent with past work, the model showed significant correlations between all four intelligence factors, including a moderate correlation between Gf and Gc $(r=.42, p<.001)$ and a large correlation between Gf and Gv $(r=.59, p<$ $.001)$.

We then specified a higher-order model to assess how the four intelligence factors load onto a higher-order $g$ factor: $\chi^{2}$ (62 df) 99.012, $p=.002$; CFI .924; RMSEA $=.057$ [90\% CI: .035, .077]; SRMR $=.066$. The model showed significant loadings of the lower-order factors onto a higher-order factor (magnitude of factor loadings in descending order): Gf (.79), Gc (.66), Gv (.62), and Gr (.60).

Next, we specified a measurement model for the three divergent thinking variables: two lab-based tasks (box and rope) and the MRI-based tasks. The four raters' creativity ratings served as indicators for their respective latent variable. Figure 3 depicts the model, which showed good fit: $\chi^{2}(51 \mathrm{df}) 78.410, p=$ .008 ; CFI .985; RMSEA $=.054$ [90\% CI: .028, .076]; SRMR $=.040$. The four raters' ratings loaded highly on the three latent variables, consistent with the high level of rater agreement and significant zero-order correlations. As expected, the two lab-based divergent thinking variables showed the largest latent correlation $(r=.64, p<.001)$. Notably, both lab-based variables showed large correlations with the MRI variable: MRI and box $(r=.55, p<.001)$ and MRI and rope $(r=.49, p<.001)$. 


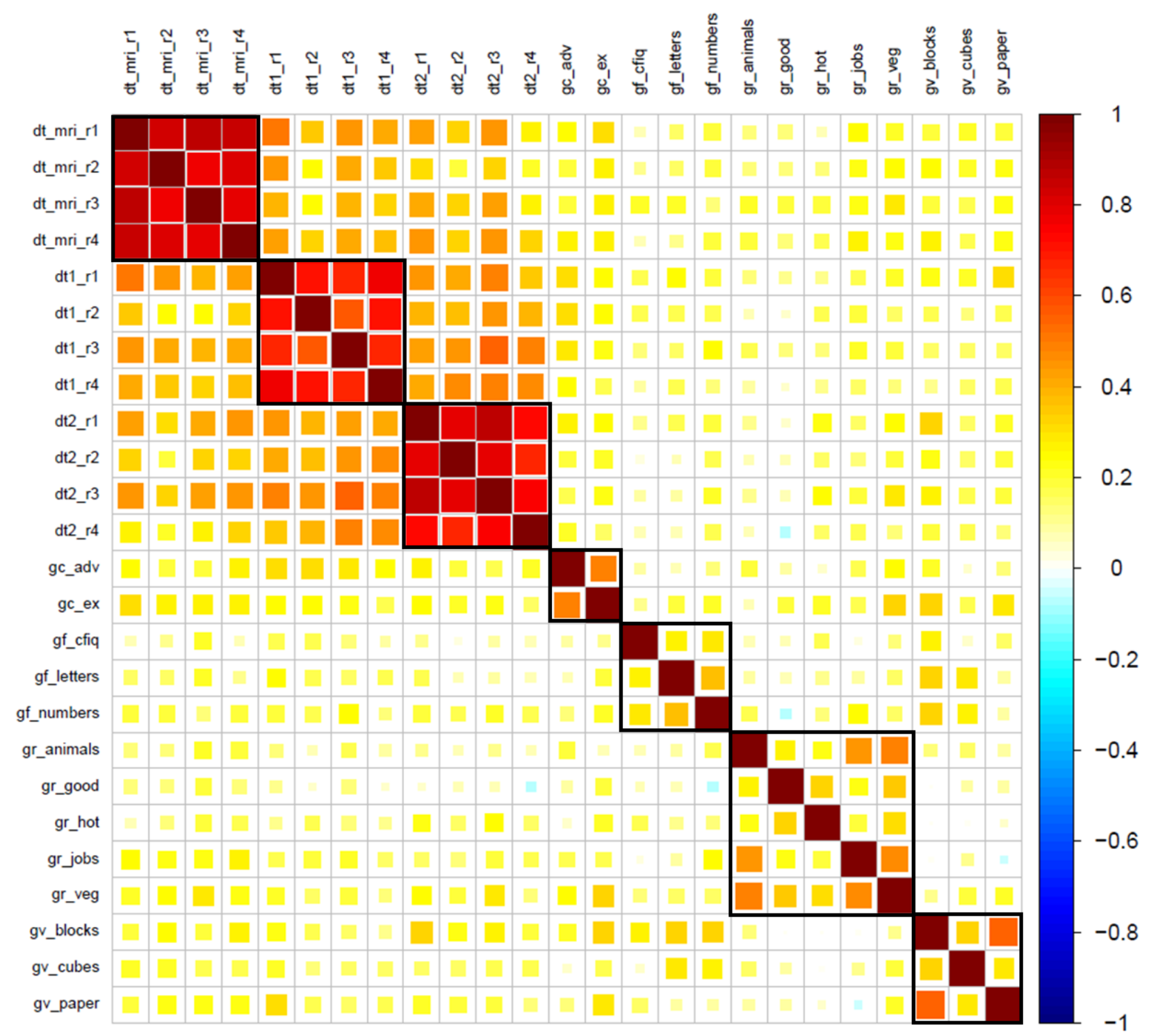

Figure 1. Pearson correlations beween all observed variables. $d t 1=$ divergent thinking, box; $d t 2=d i v e r g e n t$ thinking, rope; dt_mri = divergent thinking from MRI trials (rater 1-4); gc_adv = crystallized intelligence, advanced vocabulary; gc_ext = crystallized intelligence, extended range; gf_cfiq = fluid intelligence, Cattell Series Completion; gf_lets = fluid intelligence, letter sets; gf_nums = fluid intelligence, number series; gr_ani = broad retrieval ability, animal category fluency; gr_good = broad retrieval ability, synonyms for 'good'; gr_jobs = broad retrieval ability, occupations category; gr_hot = broad retrieval ability, synonyms for 'hot; gr_veg = broad retrieval ability, fruits and vegetables category; gv_block = visuospatial intelligence, block rotation; gv_cubes = visuospatial intelligence, cube comparison; gv_paper = visuospatial intelligence, paper folding.

Then, we specified a higher-order model to assess how the lab- and MRI-based divergent thinking variables load onto a higher-order factor: $\chi^{2}(51 \mathrm{df}) 78.410, p=.008$; CFI .985; RMSEA $=.054$ [90\% CI: $.028, .076] ;$ SRMR $=.040$. The model showed significant loadings of the lower-order factors onto a higherorder factor (magnitude of factor loadings in descending order): DT box (.84), DT rope (.76), and DT MRI (.65). 


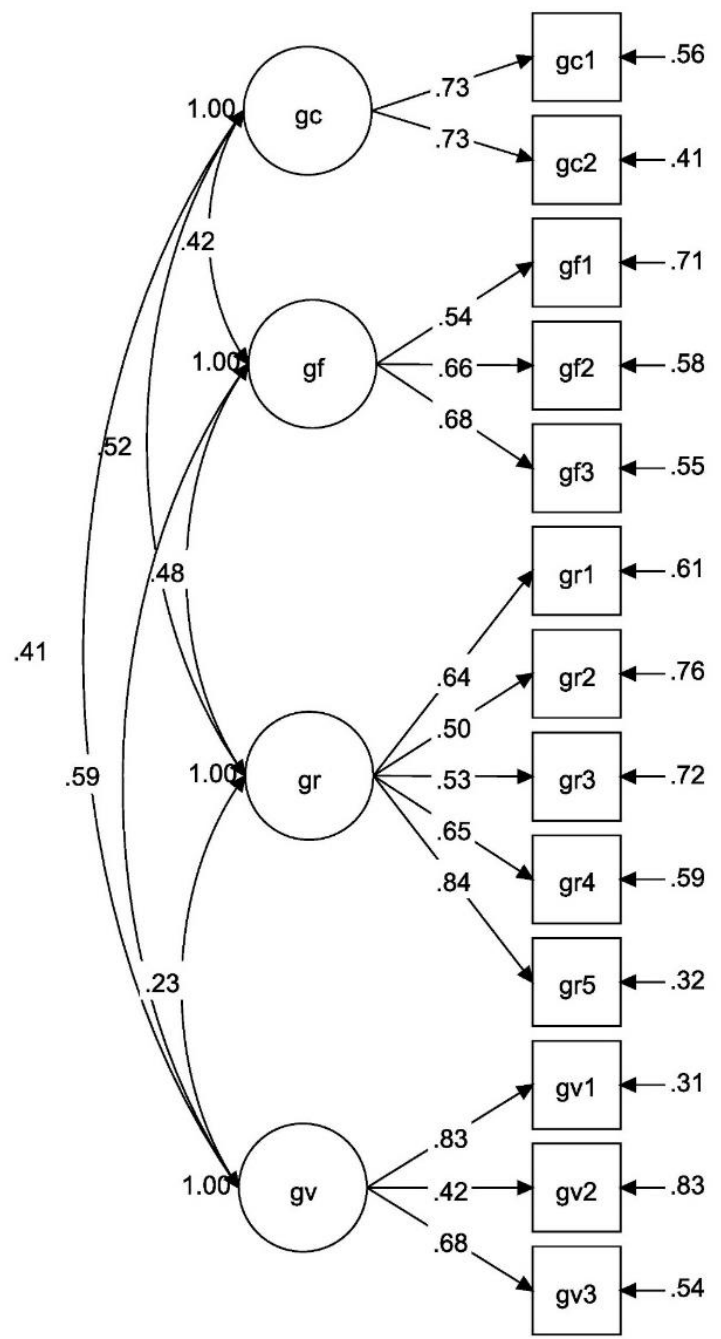

Figure 2. Confirmatory factor analysis of four intelligence facets. gc1 = crystallized intelligence, advanced vocabulary; gc2 = crystallized intelligence, extended range; $\mathrm{gf} 1$ = fluid intelligence, Cattell Series Completion; $\mathrm{gf} 2=$ fluid intelligence, letter sets; gf 3 = fluid intelligence, number series; gr1 = broad retrieval ability, animal category fluency; gr2 = broad retrieval ability, synonyms for 'good'; gr3 = broad retrieval ability, synonyms for 'hot'; gr4 = broad retrieval ability, occupations category; gr5 = broad retrieval ability, fruits and vegetables category; gv1 = visuospatial intelligence, block rotation; gv2 = visuospatial intelligence, cube comparison; gv3 = visuospatial intelligence, paper folding. $\mathrm{N}=185$.

\section{Relationships Between Divergent Thinking and Intelligence}

Having specified measurement models of intelligence and divergent thinking, we turned to assess relationships between these cognitive abilities. We began by examining latent correlations between the higher-order divergent thinking variable and the four lower-order intelligence factors. A confirmatory factor analysis showed good fit: $\chi^{2}$ (263 df) 337.831, $p=.001$; CFI .968; RMSEA = .039 [90\% CI: .025, .051]; SRMR $=.052$. The intercorrelations between the latent variables (and 95\% confidence intervals) are displayed in Table 3. The model showed significant correlations between all intelligence factors and 


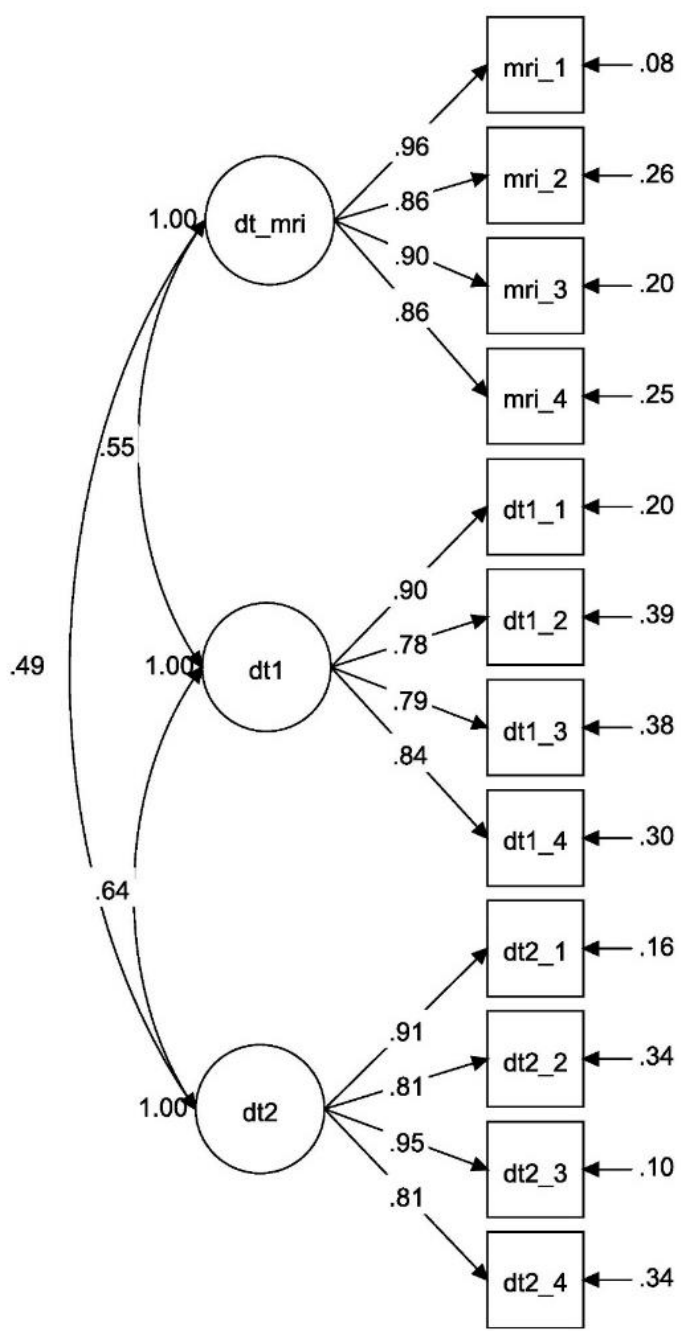

Figure 3. Confirmatory factor analysis of lab and MRI divergent thinking creativity ratings. $\mathrm{dt} 1=$ divergent thinking, box; $\mathrm{dt} 2$ = divergent thinking, rope; dt_mri = latent variable of creativity ratings from MRI trials (rater 1-4). $\mathrm{N}=186$.

divergent thinking, with magnitudes ranging from medium to large: Gr and DT, $r=.32$; Gf and DT, $r=$ .39 ; Gv and DT, $r=.41$; and Gc and DT, $r=.54$. These results replicate past work (e.g., Benedek et al., 2014; Silvia et al., 2013) and extend it by showing positive associations between divergent thinking creativity and four lower-order facets of intelligence.

Our final model assessed the latent correlation between general intelligence (i.e., higher-order $g$ ) and divergent thinking. This CFA model estimated $g$ as a higher-order factor, comprised of Gf, Gc, Gr, and Gv, serving as lower-order indicators of $g$, with the same latent divergent thinking variable as before: $\chi^{2}$ (268 df) 351.161, $p<.001$; CFI .965; RMSEA .041 [90\% CI: .028, .052]; SRMR .057. The results showed 


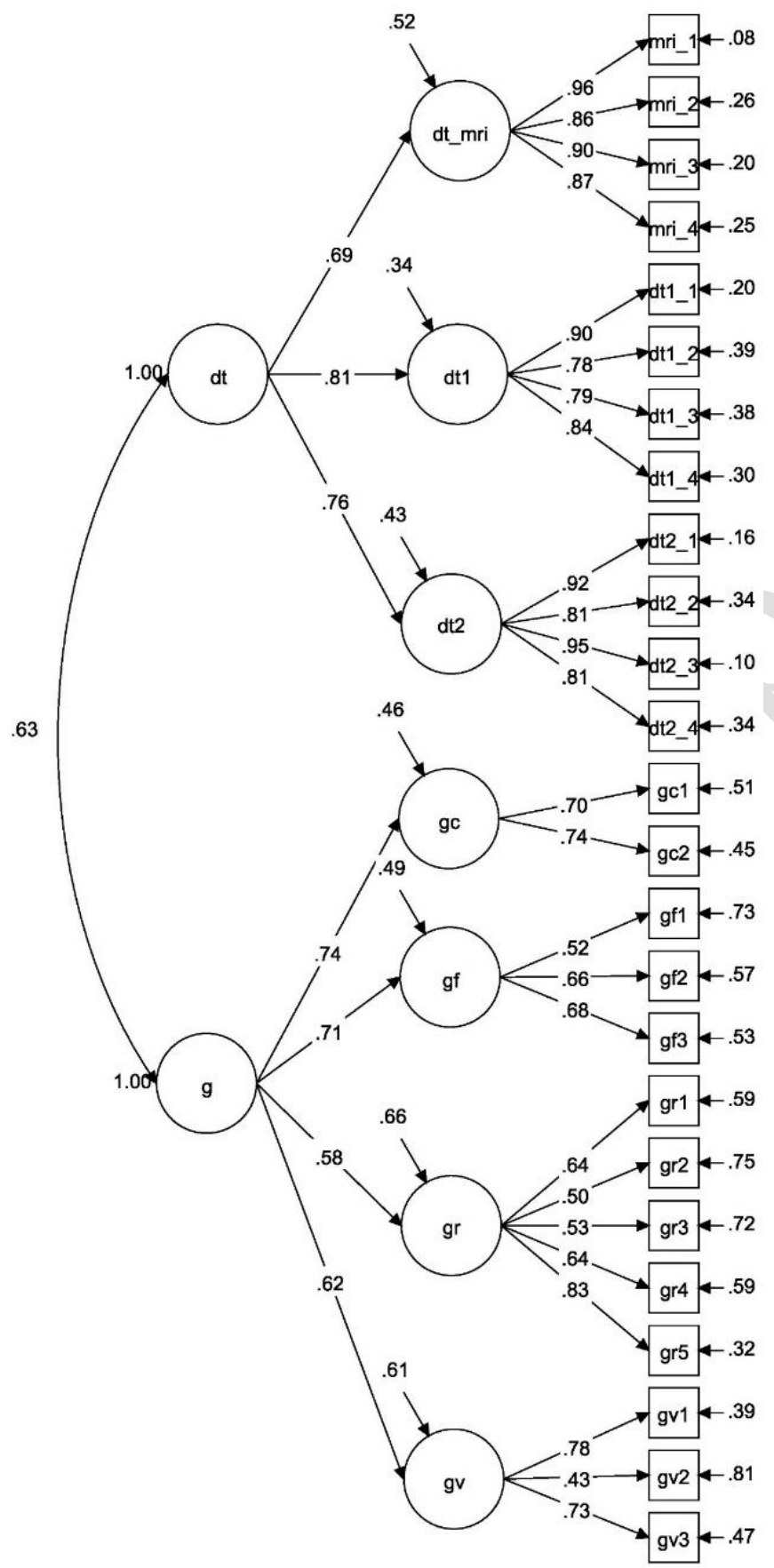

Figure 4. Confirmatory factor analysis of higher-order intelligence and divergent thinking. $\mathrm{dt} 1=$ divergent thinking, box; $\mathrm{dt} 2$ = divergent thinking, rope; $\mathrm{dt}$-mri = latent variable of creativity ratings from MRI trials (rater 1-4); $\mathrm{gc} 1$ = crystallized intelligence, advanced vocabulary; gc2 = crystallized intelligence, extended range; gf1 = fluid intelligence, Cattell Series Completion; gf2 = fluid intelligence, letter sets; gf3 = fluid intelligence, number series; gr1 = broad retrieval ability, animal category fluency; gr2 = broad retrieval ability, synonyms for 'good'; gr3 = broad retrieval ability, synonyms for 'hot'; gr4 = broad retrieval ability, occupations category; gr5 = broad retrieval ability, fruits and vegetables category; gv1 = visuospatial intelligence, block rotation; gv2 = visuospatial intelligence, cube comparison; gv3 = visuospatial intelligence, paper folding. $\mathrm{N}=186$.

a large correlation between $g$ and divergent thinking: $r=.63, p<.001$ (see Figures $4 \& 5$ ), indicating a substantial overlap between general intelligence and divergent thinking. 


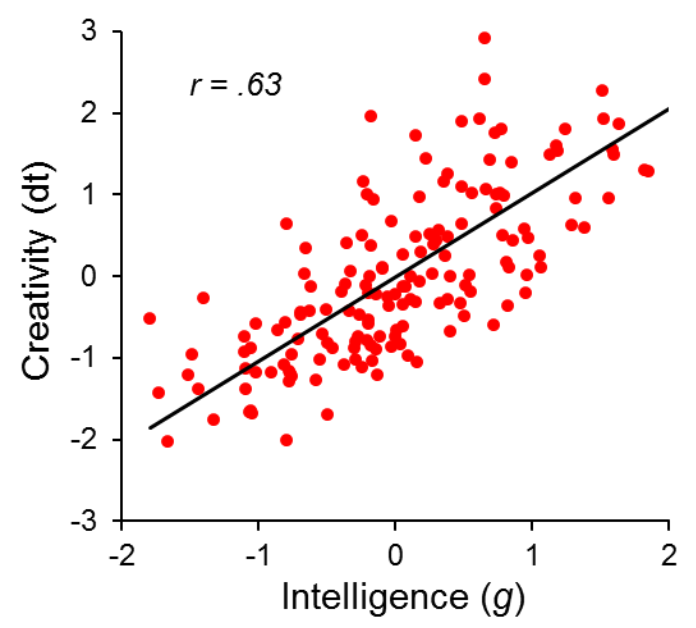

Figure 5. Scatterplot of the latent correlation between general intelligence (g) and divergent thinking (dt) creativity scores. Latent variable values are standardized for visualization. $\mathrm{N}=186$.

\section{Connectome Prediction of Divergent Thinking and Intelligence Facets}

We then examined the extent to which intelligence and divergent thinking overlap in the brain. To this end, we employed CPM to identify functional brain connections that predict divergent thinking and intelligence (both specific factors and a general factor), with the neural data consisting of functional connectivity during the divergent thinking task and the behavioral data consisting of factor scores extracted from the higher-order latent variable model (see Figure 4). We quantify the extent to which networks overlap by computing the proportion of predictive connections (edges) that appear in intelligence and divergent thinking networks (Rosenberg et al., 2018). Note that we focus on the positively correlated networks (i.e., high-ability networks) because we did not have predictions about negatively correlated networks. The following analyses use the reduced sample with fMRI data $(n=171)$.

First, we assessed prediction of individual divergent thinking ability based on functional connectivity patterns during the DT task. Replicating our prior work (Beaty et al., 2018) with a different functional brain atlas, we found a large positive correlation between the model-predicted and actual divergent thinking scores $(r=.41, p<.001)$. Beaty et al., 2018, $r=.30)$. The DT network (i.e., the overlap of all leave-one-out models) was distributed across the brain, with the highest-degree nodes (i.e., regions with more functional connections) concentrated within frontal lobes, predominantly within the executive network, as well as the visual, salience/ventral attention, and default networks (see Table 4 and Figures 6 

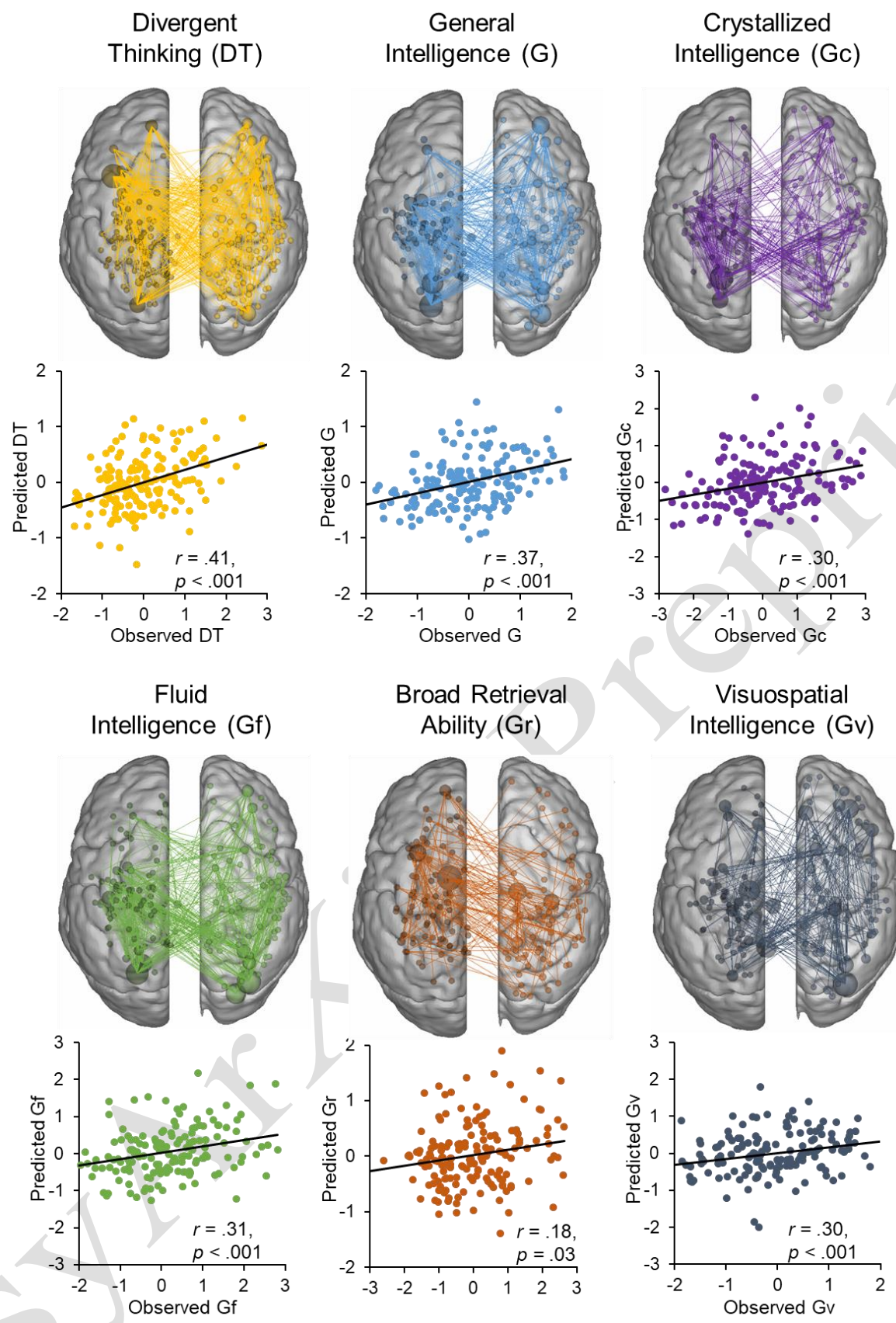

Visuospatial
Intelligence (Gv)

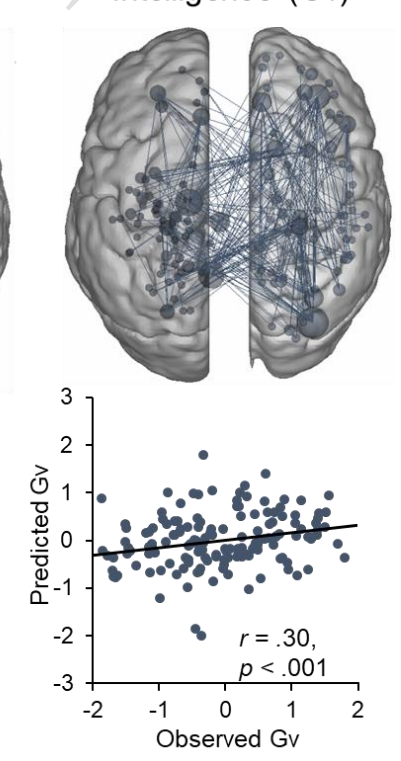

Figure 6. Functional brain networks that predict divergent thinking ability and intelligence. Observed behavior scores are latent variable factor scores extracted from the higher-order CFA (see Figure 4). Latent variable values are standardized for visualization. To further aid visualization of network connections, each network was thresholded at $2 \%$ of their total functional connections (i.e., degree). For example, the DT network included a total of 933 connections, so a $2 \%$ degree threshold applied to this network mask is 19 ; thus, nodes with at least 19 connections (k) are displayed in the DT network above. $\mathrm{N}=171$.

and 7). The highest proportion of functional connections (i.e., edges) was found between left executive control and right salience/ventral and dorsal attention networks (i.e., 9\%; see Figure 8). Participants with 

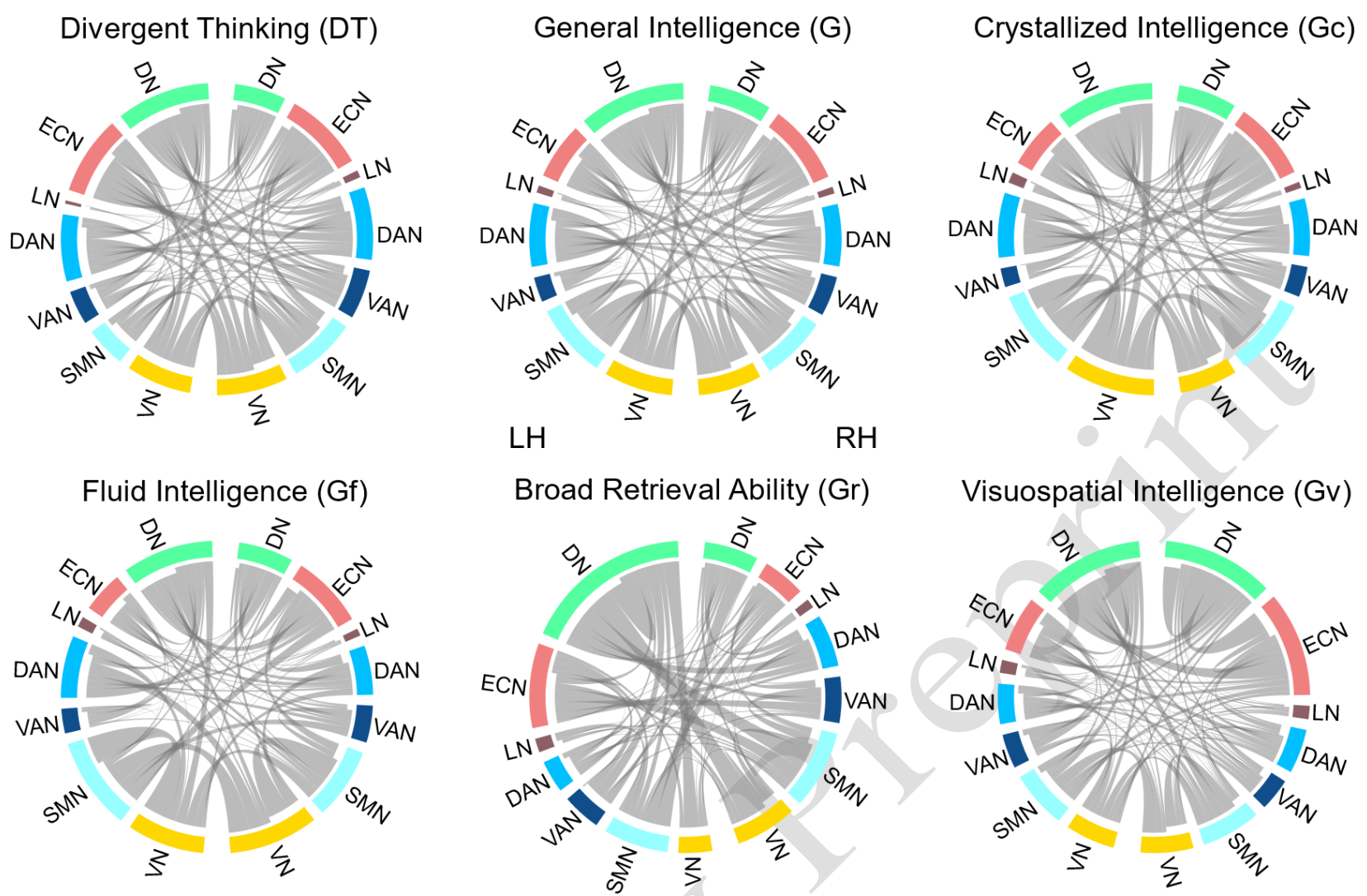

Figure 7. Connectome rings depicting functional networks that predict divergent thinking ability and intelligence. The seven networks of the Schaefer et al. (2018) atlas are labeled around the connectome rings for each hemisphere (left and right). The relative size of the colored network labels in each ring represents the relative number of edges in each network (larger labels $=$ more edges). Note that these networks correspond to the unthresholded network masks displayed in Figure 5. DAN = dorsal attention network; DN = default network; ECN = executive control network; $\mathrm{LN}=$ limbic network; SMN = sensorimotor network; VN = visual network; $\mathrm{RH}=$ right hemisphere; LH = left hemisphere; $\mathrm{N}=171$.

stronger connections in this overall network thus tended to produce more original responses on the divergent thinking assessments.

Next, we assessed the extent to which lower-order intelligence facets could be predicted from functional connectivity patterns during the DT task. Our first intelligence model examined prediction of Gc (crystallized intelligence; i.e., vocabulary knowledge). Results showed a significant correlation between the model-predicted and observed Gc scores $(r=.30, p<.001)$. High-degree nodes were found within the visual, executive, salience, somatomotor, and default networks. The highest proportion of edges (5\%) was found between the left salience/ventral attention network and the right executive network. The Gc network shared $13 \%$ functional connections with the DT network (common edges = 158); the Gc network included a total of 425 edges, so it shared $37 \%$ of its edges with the DT network. The combined DT-Gc network 


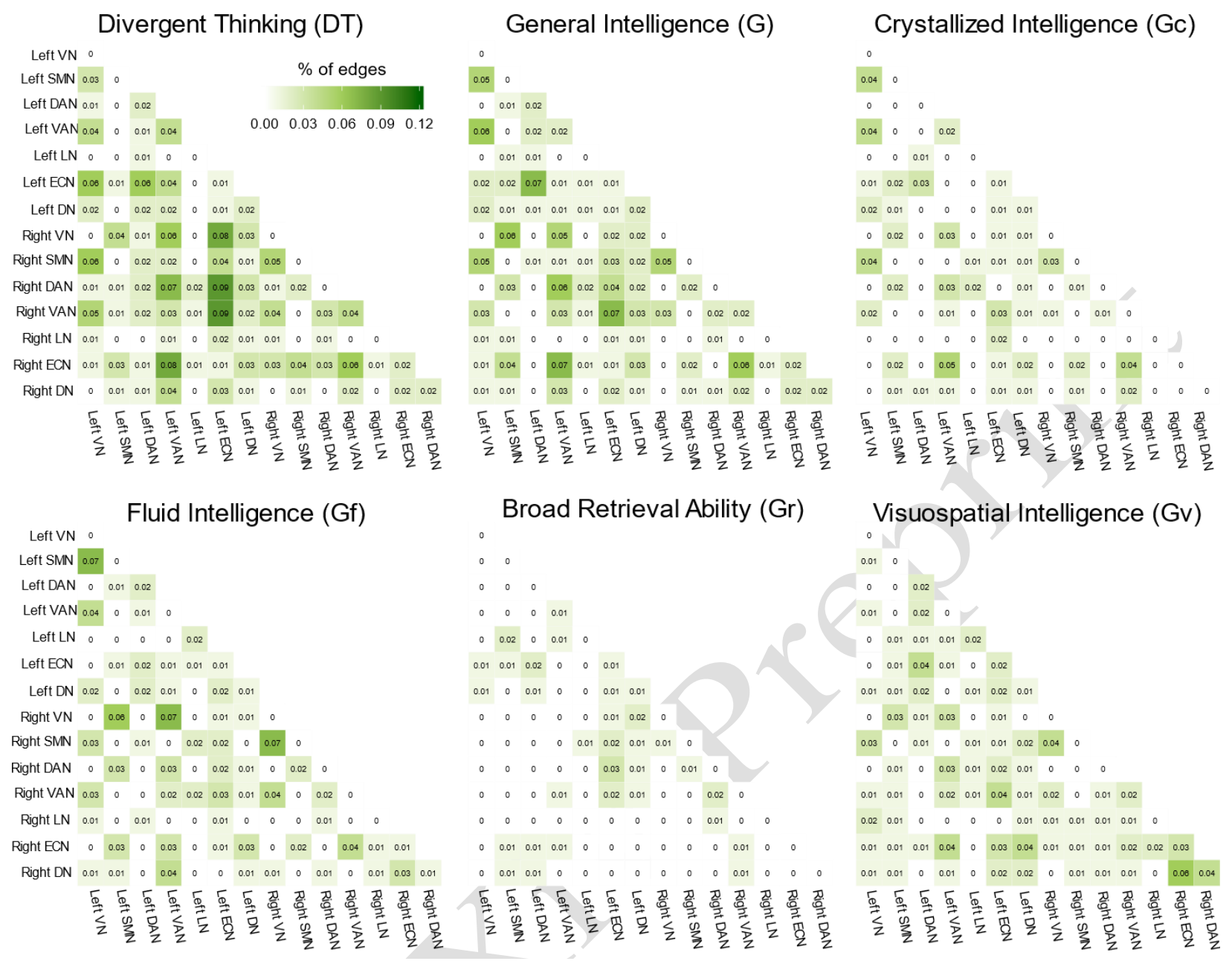

Figure 8. Proportion of edges between all pairs of networks in the divergent thinking and intelligence networks. The proportion of edges scales with the shade of green in each cell (more edges $=$ darker green). The denominator of the cells reflects the total number of edges in the respective network, and the numerator reflects the number of edges found between a given network pair. The seven functional networks of the Schaefer et al. (2018) atlas are listed for both hemispheres along the $\mathrm{x}$ - and $\mathrm{y}$-axes. DAN $=$ dorsal attention network; $\mathrm{DN}=$ default network; $\mathrm{ECN}=$ executive control network; $\mathrm{LN}=$ limbic network; $\mathrm{SMN}=$ sensorimotor network; $\mathrm{VN}=$ visual network; $\mathrm{RH}=$ right hemisphere; $\mathrm{LH}=$ left hemisphere; $\mathrm{N}=171$.

consisted of high-degree nodes within executive, visual networks, and salience/ventral attention (see Table 5), with the highest proportion of common edges found between the left salience/ventral attention network and the right control network (5\%). Figures 9 and 10 depict the combined overlap of functional connections and the proportion of common connections, respectively. Figure 11 visualizes the overlap of the DT network with all intelligence networks, as well as the overlap of all intelligence networks.

We then examined prediction of Gf (fluid intelligence) based on functional connectivity patterns during the DT task. Results showed a significant correlation between the model-predicted and latent Gf score $(r=.31, p<.001)$. Similar to the Gc network, several of the highest-degree nodes were found within 

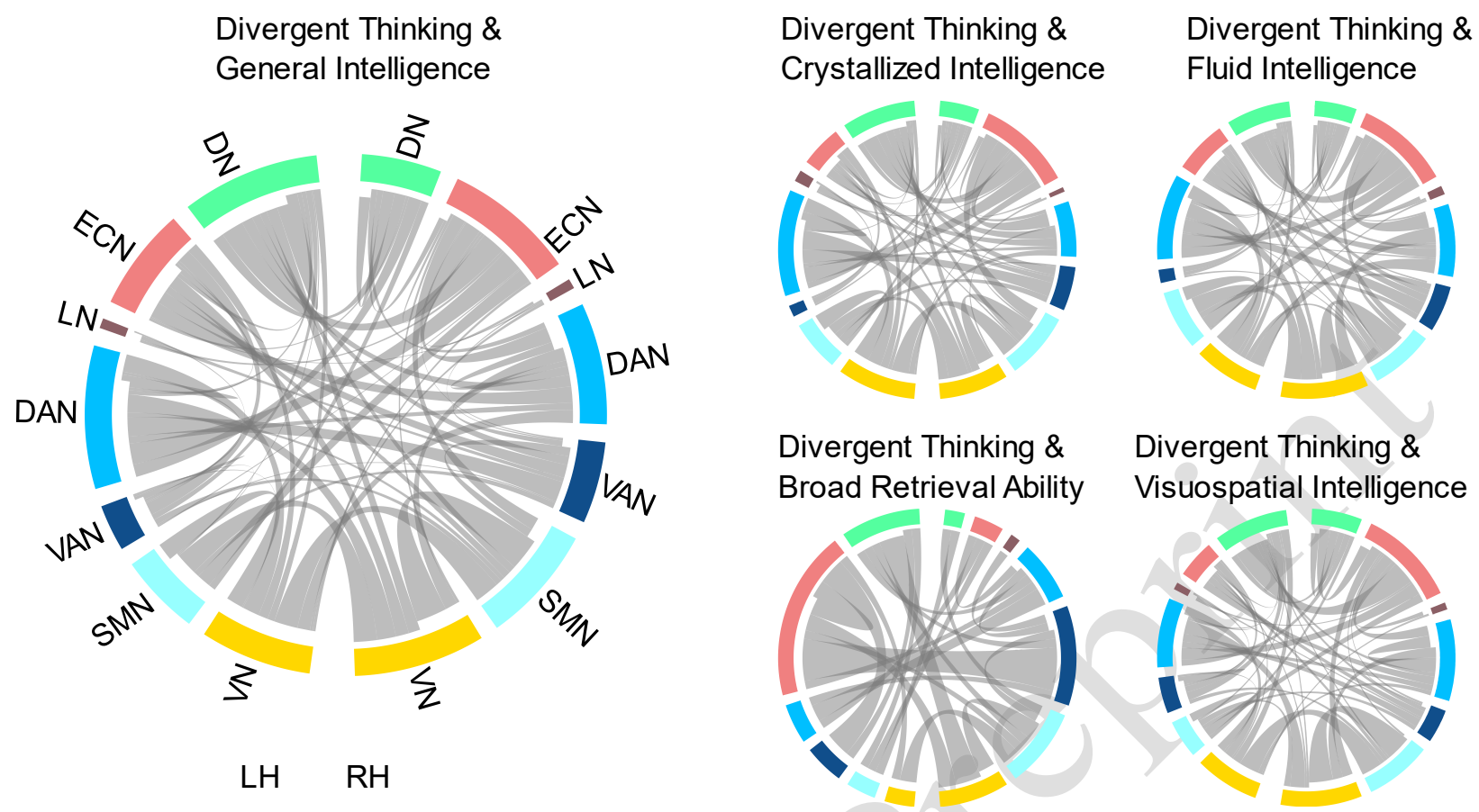

Figure 9. Connectome rings depicting the overlap of functional networks that predict divergent thinking ability and intelligence. Network overlap masks were computed by multiplying the binary cells of the divergent thinking and intelligence network masks shown in Figure 7. The seven networks of the Schaefer et al. (2018) atlas are labeled around the connectome rings for each hemisphere (left and right). The relative size of the colored network labels in each ring represents the relative number of edges in each network (larger labels $=$ more edges). Note that these networks correspond to the unthresholded network masks displayed in Figure 6. DAN = dorsal attention network; DN = default network; $\mathrm{ECN}$ = executive control network; $\mathrm{LN}=$ limbic network; $\mathrm{SMN}=$ sensorimotor network; $\mathrm{VN}=$ visual network; $\mathrm{RH}=$ right hemisphere; $\mathrm{LH}=$ left hemisphere; $\mathrm{N}=171$.

the visual network, with additional high-degree nodes distributed across the executive, salience/ventral attention, dorsal attention, and default networks. The highest proportion of edges (7\%) was between the left salience/ventral attention and right visual networks, among other networks. The Gf network shared 12\% of functional connections with the DT network (common edges = 170); the Gf network included a total of 617 edges, so it shared $28 \%$ of its connections with the DT network. The combined DT-Gf network consisted of high-degree nodes within executive networks, salience/ventral attention, visual, and dorsal attention networks, with the highest proportion of edges (2\%) found between the left salience/ventral attention several networks, including right executive control, right dorsal attention, and right visual networks, among other networks (see Figure 10).

Next, we examined prediction of Gr (broad retrieval ability) based on functional connectivity patterns during the DT task. Results showed a small correlation between the model-predicted and latent Gr 

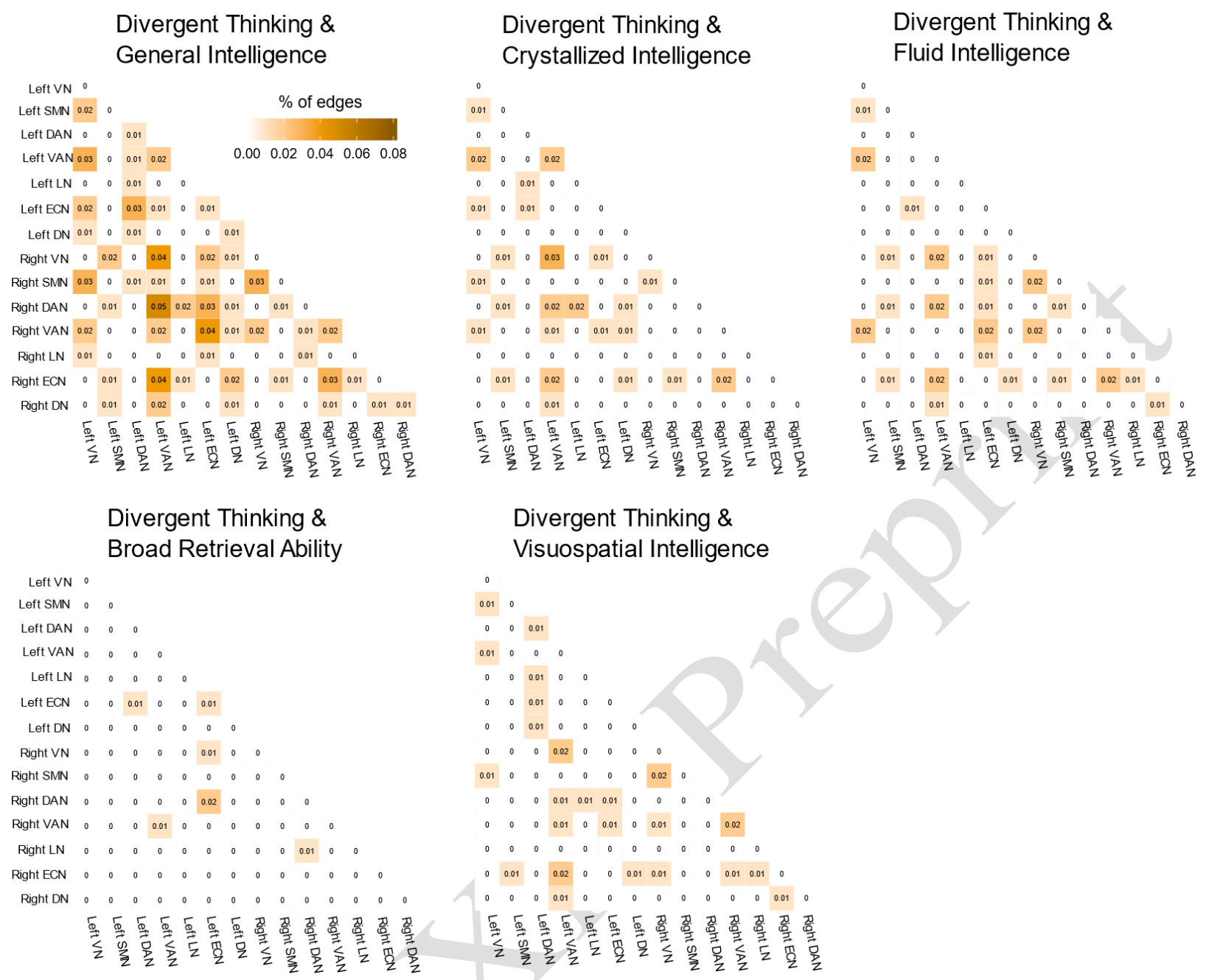

Figure 10. Proportion of edges between all pairs of networks in the combined divergent thinking and intelligence networks. The proportion of edges scales with the shade of green in each cell (more edges $=$ darker orange). The denominator of the cells reflects the total number of edges in the respective network, and the numerator reflects the number of edges found between a given network pair. The seven functional networks of the Schaefer et al. (2018) atlas are listed for both hemispheres along the $\mathrm{x}$ - and $\mathrm{y}$-axes. DAN = dorsal attention network; DN = default network; ECN = executive control network; $\mathrm{LN}=$ limbic network; SMN = sensorimotor network; VN = visual network; RH = right hemisphere; $\mathrm{LH}=$ left hemisphere; $\mathrm{N}=171$.

score $(r=.18, p=.03)$. The highest-degree nodes were found within the control network, with other highdegree nodes distributed across the salience/ventral attention, default, dorsal attention, and somatomotor networks. The highest proportion of edges (3\%) was between the left control and right dorsal attention networks. The Gr network shared 3\% of functional connections with the DT network (common edges = 37); the Gr network included a total of 244 edges, so it shared $15 \%$ of its connections with the DT network. The combined DT-Gr network consisted of high-degree nodes within control, default, salience/ventral 

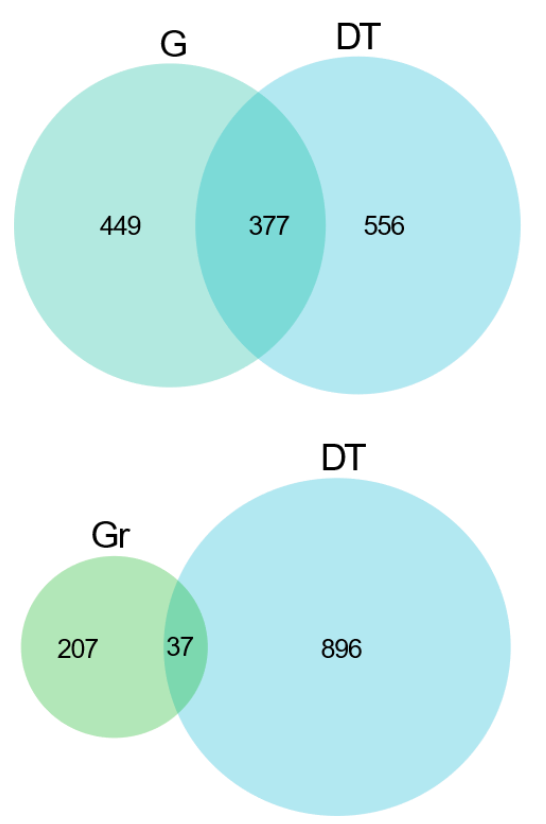

Figure 11. Venn diagrams depicting the number of common and unique edges between divergent thinking and intelligence networks. The colored circles represent networks predictive of divergent thinking and intelligence. The number of edges in the overlapping circle regions corresponds to the number of edges in the combined networks depicted in Figures 9 and 10. Circle size corresponds to the relative number of edges in the network.

attention, and somatomotor networks, with the highest proportion of edges found between the left control and right dorsal attention networks $(3 \%)$.

We then assessed prediction of Gv (visuospatial intelligence) based on functional connectivity patterns during the DT task. Results showed a significant correlation between the model-predicted and latent Gv score $(r=.30, p<.001)$. Similar to the Gf and Gc networks, high-degree nodes were found within the visual network and executive networks; the Gv network also included several high-degree nodes within the default network (see Table 4). The highest proportion of edges (6\%) was between the right executive control and right default networks (see Figure 8). The Gv network shared $11 \%$ of its functional connections with the DT network (common edges $=150$ ); the Gv network included a total of 547 edges, so it shared $27 \%$ of its connections with the DT network. The combined DT-Gv network showed high-degree nodes within control, salience/ventral attention, somatomotor, and visual networks, with the highest proportion of edges (2\%) found between left salience/ventral attention and right executive and right visual networks, among others. 
Finally, we examined prediction of higher-order $g$ (general intelligence) based on functional connectivity patterns during the DT task. Results showed a significant correlation between the modelpredicted and latent $g$ score $(r=.37, p<.001)$. The $g$ network included high-degree nodes within control, salience/ventral attention, dorsal attention, and visual networks, with the highest proportion of edges (7\%) found between bilateral salience/ventral attention and control networks, as well as left dorsal attention and left executive control networks. The $g$ network shared $27 \%$ of its functional connections with the DT network (common edges $=377$ ); the $g$ network included a total of 826 edges, so it shared $46 \%$ of its connections with the DT network. Regarding anatomy, the high-degree nodes of the combined DT- $g$ network were mostly concentrated within the control network, as well as visual, dorsal attention, and salience/ventral attention networks (see Table 5). The highest proportion of edges (5\%) was found between left salience/ventral attention and right dorsal attention networks; the combined network also showed common connections between salience/ventral attention and executive control networks (see Figure 10). General intelligence and divergent thinking thus showed overlapping functional connections between brain networks associated with the executive control of attention and cognition.

\section{Discussion}

The present research combined brain-based prediction and psychometric tools to examine the extent to which creative cognition and intelligence overlap in brain and behavior. At the behavioral level, we found that four intelligence facets (Gc, Gf, Gr, and Gv) positively correlated with divergent thinking, with effect sizes ranging from moderate to large. Modeling these intelligence facets as indicators of a higher-order factor revealed a large latent correlation between $g$ and divergent thinking $(r=.63)$. At the neural level, connectome-based predictive models built on fMRI data during a divergent thinking task showed reliable prediction of both divergent thinking and intelligence facets, revealing considerable overlap in the functional connectivity patterns that predict both cognitive abilities, predominantly within executive control, salience/ventral attention, and visual networks. Taken together, these results provide new insight into the nature of the creative cognition-intelligence relationship by mapping behavioral associations to functional brain network connections. 
A latent variable model yielded positive correlations between divergent thinking and controlled semantic retrieval (Gr), higher-order reasoning (Gf), vocabulary knowledge (Gc), and mental manipulation of spatial stimuli $(\mathrm{Gv})$, with GC showing the largest correlation with divergent thinking. Gc involves utilizing and manipulating acquired knowledge during the problem-solving process (Cattell, 1963). Thus, our extension of work linking Gc and creativity (Cho et al., 2010) is perhaps not surprising, as Gc may permit access to a more extensive information "toolbox" stored in memory. Solving an ill-defined divergent thinking task requires people to employ various mental strategies that rely on broad lexical access, activation of previously acquired declarative (knowing "what") and procedural (knowing "how") knowledge and relevant experiences (Hunt, 2000). When presented with an unfamiliar and open-ended task, one's depth and breadth of knowledge may be fundamental to producing novel solutions that go beyond the known meaning of the target concept (Basadeur \& Gelade, 2005). In our study, participants were required to generate creative uses for common objects (Guilford, 1967). Here, Gc may facilitate divergent ideation by allowing people to consider objects relative to past experiences, reflect upon knowledge schemas, and evaluate the appropriateness of accessible information (Amabile, 2018).

We also observed positive correlations between the intelligence facets $\mathrm{Gr}$, Gf, Gv, and divergent thinking. Gr involves accessing facts and conceptual knowledge from long-term memory (Silvia et al., 2013). Previous work has suggested that such controlled semantic retrieval may facilitate an associative process in which novel ideas are generated by combining distantly-related concepts in semantic memory (Abraham, 2014; Abraham \& Bubic, 2015; Beaty et al., 2020; Bowden \& Jung-Beeman, 1998; Green, 2016; Mednick, 1962). It is possible that the correlation between Gr and divergent thinking in our sample reflects an ability to conceptually expand the meaning of AUT prompts, such that unexpected, original, and taskappropriate concepts are accessed as viable response candidates during divergent thinking (Abraham, 2014). Given Gr's role in controlled semantic retrieval, Gr could also support divergent thinking by generating and executing complex retrieval cues to strategically guide the search process during divergent thinking (cf., Silvia et al., 2013). 
Gf involves pattern and rule discernment as well as the controlled selection and implementation of strategies to solve novel tasks (McGrew, 2009; Silvia, 2008). Evidence for the role of Gf in creative cognition indicates that higher-order reasoning may support an ability to flexibly shift from less effective to more effective problem-solving approaches (Nusbaum \& Silvia, 2011; Silvia, 2015). Because divergent thinking is an effortful cognitive process that requires executive inhibition of standard responses to produce unconventional responses (Silvia \& Beaty, 2012), the Gf-creativity association observed in the present experiment adds to existing research which suggests that executive control and higher-order reasoning support the quality of divergent ideas (Benedek et al. 2012). Gv involves mentally manipulating threedimensional stimuli. Although less explored in the creativity literature, visuospatial intelligence may be particularly important for problem-solving during divergent tasks that require imaginal motor planning (Aziz-Zadeh, Liew, \& Dandekar, 2013). The AUT requires that individuals generate uses for common objects; therefore, idea generation may integrate mental simulations of possible utility functions. Gv may initially support mental simulation of common uses to scaffold imagined novel uses, such as envisioning ways to tie rope to make a handle for a drawer, or to fashion a portable wrestling ring. Continued creativity research should further explore individual differences in Gv and task conditions that integrate visuospatial elements (e.g., drawing), which may offer additional interpretations of the Gv-divergent thinking link.

Regarding general intelligence, a second latent variable model showed a large correlation between $g$ and divergent thinking. Specifically, when $g$ was modeled as a higher-order factor indicated by the four lower-order facets, over $40 \%$ of the variance in divergent thinking ability could be explained. Notably, the large effect sizes reported here are broadly consistent with those reported elsewhere using latent variable models, but they are notably larger than earlier work reporting zero-order correlations (e.g., Kim, 2005). We focused on subjective assessments of the creative quality of divergent thinking responses (Benedek, Mühlmann, Jauk, \& Neubauer, 2013; Silvia et al., 2008), which typically yield stronger relationships with intelligence than fluency- and uniqueness-based metrics (Silvia, 2015). We also used two modes of divergent thinking assessment—-many brief trials in the scanner coupled with conventional computer-based trials - providing a comprehensive measurement approach that extends past behavioral work using a small 
number of trials and cues to assess divergent thinking (cf. Barbot, 2018). Taken together, the current study suggests that the intelligence-creative cognition relationship is robust when latent variable models are combined with multiple assessments of each cognitive ability.

Our study additionally provides a first look at how intelligence and creative cognition overlap within the brain's functional connectome. Using a data-driven prediction approach, CPM, we compared functional connectivity that predicted divergent thinking and intelligence facets. By overlaying these functional connectivity maps, we identified which connections contributed to the prediction of both cognitive abilities. Extending our previous CPM study (Beaty et al., 2018) with a different functional atlas, we found that divergent thinking ability could be predicted based on the strength of functional connectivity between executive, salience, and default network nodes (as well as other networks), despite some differences between this analysis and our prior study (i.e., brain atlas and preprocessing pipeline).The anatomical location and concentration of predictive nodes and connections differed to some extent from our prior study (Beaty et al., 2018), consistent with expected variation from employing a new brain atlas and preprocessing pipeline (i.e., fMRIprep). Extending our previous work, we found that individual intelligence facets could be predicted based on patterns of functional connectivity during divergent thinking, and these patterns shared considerable (but variable) overlap with those associated with divergent thinking performance. As expected, the magnitude of the CPM effect size was largest for divergent thinking, with smaller but moderate effects for the intelligence variables. Notably, our previous study found that functional connectivity strength within a "high-creative" network did not correlate with fluid intelligence in one of the external validation analyses using resting-state fMRI data, providing evidence for the specificity of this network in predicting divergent thinking with task-based fMRI data. The current study extends our previous work by modeling separate predictive brain networks for divergent thinking and several facets of intelligence (along with a general factor), quantifying the degree of overlap in the predictive features (connections) within these networks.

The present findings are consistent with recently hypothesized distinctions between the definitions of intelligence and creativity (Jung \& Chohan, 2019). On this view, intelligence is defined as a function of 
cognitive processes that support rapid and accurate problem-solving, whereas creativity is defined as a function of cognitive processes that support the combination of novelty and utility. Although there are obvious differences in the operationalization of these constructs, evidence points to the confirmation of individual differences in intelligence and creativity to two adaptive behavioral axes: exploratory (e.g., rapid and novel) and restraint (e.g., accurate and useful; Jung \& Chohan, 2019). Notably, aspects of intelligence and creativity are captured by both axes, as intelligence is characterized by rapid (exploratory) and accurate (restraint) thought, whereas creativity is characterized by novel (exploratory) and useful (restraint) thought. Furthermore, these complementary axes correspond to large-scale brain networks - the executive network and default network, respectively (Basten, Stelzel, \& Fiebach, 2013; Beaty et al., 2016; Jung et al., 2013; Jung \& Chohan, 2019) — which is consistent with our findings that both networks are relevant to abilities characteristic of intelligence and creative cognition.

Comparing the lower-order/specific intelligence facet maps with the divergent thinking map revealed high-degree nodes within executive control, salience/ventral attention, and visual networks. The executive control network plays a key role in the manipulation of information in working memory, inhibition of prepotent responses, and maintenance of higher-order goals during task execution (Niendam et al., 2012). This network has been consistently implicated in studies of creativity and intelligence (Vartanian, 2018). The salience/ventral attention network is associated with the detection of behaviorallyrelevant information — both within the environment and internally — and it plays a central role in switching between the default and executive control networks (Uddin, 2015). In the context of creative cognition, the salience network may facilitate dynamic switching between idea generation (default network) and idea evaluation (control network; Beaty et al., 2016, 2019). Thus, stronger connections between salience and control networks found in the current study may reflect a greater capacity of more intelligent participants to engage one component of this switching mechanism, potentially in the service of idea evaluation or other control-relevant processes relevant for creative cognition (e.g., switching or inhibition).

Interestingly, the CPM also consistently implicated the visual network. Although the role of the visual network is less well-documented in creativity neuroscience, in light of related past work, we suspect 
that the visual network may play a role in mental imagery processes. The visual network shows consistent activation during tasks that involve "mental time travel"-facilitating episodic recall in service of prospective, episodic future-thinking (Benedek et al., 2014b) — which may foster imagining possible actionbased simulations of alternative uses for a stimulus. Future work should examine whether visual cortices contribute to performance on other divergent thinking tasks, such as figural or musical creative ability, which allow physical modification of the task space (e.g., via assembling notes or sketching ideas). On the one hand, such physical manipulation of task stimuli that could attenuate reliance on the visual/imagery system for ideation by incorporating spatial and physical modalities; on the other hand, it could amplify reliance on the visual system by increasing the saliency and perceived applicability of imagined episodes, as they are actively explored in real time.

Across all comparisons of lower-order facets and divergent thinking, the prefrontal cortex of the control network consistently emerged as one of the most predictive brain regions. This observation is consistent with previous research implicating the lateral prefrontal cortex (DLPFC) in intelligence and creative cognition (Barbey et al., 2013; Chen et al., 2016; Jung et al., 2010), likely due to their common reliance on cognitive control (Benedek et al., 2014). The control network may play an important role in creative idea evaluation (Kleinmintz et al., 2019), particularly at later stages of idea production (Beaty et al., 2015), by determining whether candidate ideas fit the constraints of creative task demands. Considered in the context of the current findings, the common prediction of intelligence facets (Gc, Gf, Gr, and Gv) and divergent thinking within the control network may reflect more efficient deployment of goal-directed and strategic cognitive processes during creative task performance.

Regarding general intelligence and creative cognition, CPM showed high-degree nodes consistent with lower-order models, predominantly within executive control, salience/ventral attention, and visual networks. Interestingly, the general intelligence network shared nearly half of its functional connections with the divergent thinking network (i.e., 46\%), in line with the large behavioral effect of $g$ on divergent thinking performance. The highest degree nodes in this combined network were located with bilateral visual cortex and lateral prefrontal regions of the control network. Similar to the lower-order intelligence CPM, 
the combined DT-g network included functional connections linking control network with salience/ventral attention networks, as well as dorsal attention and visual networks. This finding extends previous research reporting functional connectivity between executive and salience networks, in turn suggesting that these connections may be stronger in more intelligent people. Intelligence may therefore contribute to creative cognition by engaging executive control processes to strategically search memory, inhibit common response tendencies, combine concepts, manipulate mental images, and evaluate the utility of candidate ideas (Beaty et al., 2016). Taken together, the present work provides clarity on the complex relationship between intelligence and creativity by linking specific cognitive abilities to specific functional connections that predict a person's ability to think creatively.

\section{Summary, Limitations, and Future Directions}

Our findings contribute to the growing literature highlighting the role of intelligence to creative cognition (Benedek et al., 2018; Christensen et al., 2018; Forthmann et al., 2019; Karwowski et al., 2016; Silvia, 2015). We found a strong correlation between $g$ and divergent thinking, as well moderate to large correlations between lower-order facets and divergent thinking, pointing to shared common processes underlying these cognitive abilities. Looking ahead, these findings could be extended by examining simultaneous effects of lower-order facets and higher-order $g$ using bifactor modeling. Past research has reported joint effects of lower-order facets and $g$ on related creative thinking abilities, such as humor production (Christensen et al., 2018). The sample size of the current study did not permit successful bifactor modeling (fit indices were inadequate), but we encourage future work to leverage bifactor models to this end. Further research is also needed to delineate whether the effects of lower-order facets and higher-order $g$ extend beyond laboratory-based assessments of divergent thinking measures to contribute to a broader range of creative domains, including musical and artistic improvisations or creative writing ability, for instance.

Extensions of this work should also aim to investigate a more heterogenous sample, as $69 \%$ of our participants were female, and some previous work has demonstrated that the brain correlates of both intelligence and creativity relates to sex (Abraham, 2016; Deary, Pink, \& Johnson, 2010; Ryman et al., 
2014). Notably, however, sex differences are not ubiquitous in the literature in either domain (see Baer \& Kaufman, 2008; Colom, 2000; Halpern \& LaMay, 2000; Miller \& Halpern, 2014; Pagnani, 2011), and it is important to highlight that sex differences in cognitive abilities may emerge as a function of strategies employed during the ideational process, as opposed to reflecting differential ability (Abraham, 2014, 2016; Deary, Pink, \& Johnson, 2010). For example, Abraham and colleagues (2014) observed greater recruitment of brain regions associated with outcome-focused decision making, semantic memory, and rule learning during divergent thinking assessed via the AUT in men, whereas greater neural activation was observed in regions associated with language comprehension and perception of social cues among women. Notably, there were no sex differences in behavioral originality performance, which provides support for the possibility that sex differences in brain activity may predominantly reflect the adoption of different strategies to achieve similar outcomes; however, this topic warrants further exploration.

In addition, a broader issue in the field of cognitive neuroscience lies in the inherently complex mapping of cognitive processes onto brain regions, which often fails to reflect a one-to-one mapping (Krakauer, 2017). Although two cognitive processes may share the same neural mechanisms, these processes may actually be distinct, with different computations performed within the same brain region (Katz, 2016). Conversely, two cognitive processes may engage different neural mechanisms and yet be very similar, which indicates similar computations performed in different brain regions (Marder \& Goaillard, 2006). Given this understanding, the question of whether intelligence and creative cognition hinge on similar cognitive processes is far from resolved, and future behavioral and neuroscientific research is warranted to disentangle interpretations of spatial overlap, which could further inform psychological theories of intelligence and creativity.

Our findings indicate that similar functional brain networks support creative cognition and intelligence. We used functional imaging data acquired during divergent thinking to identify functional connections that contribute to the prediction of both cognitive abilities. This approach extends recent applications of brain-based prediction approaches (e.g., CPM) by mapping cognitive factors to specific brain regions and connections within complex patterns of functional connectivity. Although task-based 
fMRI data improve prediction of human cognitive abilities compared to task-free data (Greene et al., 2018), it is worth noting that our connectome-based models were built only on divergent thinking data but predicted both divergent thinking and intelligence. Thus, extending our work, future research could combine functional connectivity data obtained from both cognitive domains to directly compare their overlap. Such approaches can provide increasingly higher resolution on the cognitive and neural mechanisms of complex human abilities like intelligence and creative cognition. 


\section{References}

Abraham, A. (2014). Creative thinking as orchestrated by semantic processing vs. cognitive control brain networks. Frontiers in Human Neuroscience, 8, 95.

Abraham, A. (2016). Gender and creativity: An overview of psychological and neuroscientific literature. Brain Imaging and Behavior, 10, 609-618.

Abraham, A. (2018). The neuroscience of creativity. Cambridge University Press.

Abraham, A., \& Bubic, A. (2015). Semantic memory as the root of imagination. Frontiers in Psychology, 6, 325.

Abraham, A., Thybusch, K., Pieritz, K., \& Hermann, C. (2014). Gender differences in creative thinking: behavioral and fMRI findings. Brain Imaging and Behavior, 8, 39-51.

Amabile, T. M. (2018). Creativity in context: Update to the social psychology of creativity. Routledge.

Ardila, A., Ostrosky-Solís, F., \& Bernal, B. (2006). Cognitive testing toward the future: The example of semantic verbal fluency (ANIMALS). International Journal of Psychology, 41, 324-332.

Avants, B. B., Epstein, C. L., Grossman, M., \& Gee, J. C. (2008) Symmetric Diffeomorphic Image Registration with Cross-Correlation: Evaluating Automated Labeling of Elderly and Neurodegenerative Brain. Medical Image Analysis, 12, 26-41.

Avitia, M. J., \& Kaufman, J. C. (2014). Beyond $g$ and $c$ : The relationship of rated creativity to long-term storage and retrieval (Glr). Psychology of Aesthetics, Creativity, and the Arts, 8, 293-302.

Aziz-Zadeh, L., Liew, S. L., \& Dandekar, F. (2013). Exploring the neural correlates of visual creativity. Social Cognitive and Affective Neuroscience, 8(4), 475-480.

Baer, J., \& Kaufman, J. C. (2008). Gender differences in creativity. The Journal of Creative Behavior, 42, $75-105$.

Barbey, A. K. (2018). Network neuroscience theory of human intelligence. Trends in Cognitive Sciences, 22, 8-20.

Barbey, A. K., Colom, R., \& Grafman, J. (2013). Architecture of cognitive flexibility revealed by lesion mapping. NeuroImage, 82, 547-554. 
Barbey, A. K., Colom, R., \& Grafman, J. (2013). Dorsolateral prefrontal contributions to human intelligence. Neuropsychologia, 51, 1361-1369.

Barbot, B. (2018). The dynamics of creative ideation: Introducing a new assessment paradigm. Frontiers in Psychology, 9, 2529.

Barron, F., \& Harrington, D. M. (1981). Creativity, intelligence, and personality. Annual Review of Psychology, 32, 439-476.

Basadur, M., \& Gelade, G. A. (2005). Modelling applied creativity as a cognitive process: Theoretical foundations. Korean Journal of Thinking and Problem Solving, 15, 13.

Basten, U., Stelzel, C., \& Fiebach, C. J. (2013). Intelligence is differentially related to neural effort in the task-positive and the task-negative brain network. Intelligence, 41, 517-528.

Beaty, R. E., Benedek, M., Kaufman, S. B., \& Silvia, P. J. (2015). Default and executive network coupling supports creative idea production. Scientific Reports, 5, 10964.

Beaty, R. E., Benedek, M., Silvia, P. J., \& Schacter, D. L. (2016). Creative cognition and brain network dynamics. Trends in Cognitive Sciences, 20, 87-95.

Beaty, R. E., Benedek, M., Wilkins, R. W., Jauk, E., Fink, A., Silvia, P. J., ... \& Neubauer, A. C (2014a). Creativity and the default network: A functional connectivity analysis of the creative brain at rest. Neuropsychologia, 64, 92-98.

Beaty, R. E., Chen, Q., Christensen, A. P., Kenett, Y. N., Silvia, P. J., Benedek, M., \& Schacter, D. L. (2020). Default network contributions to episodic and semantic processing during divergent creative thinking: A representational similarity analysis. NeuroImage, 209, 116499.

Beaty, R. E., Kenett, Y. N., Christensen, A. P., Rosenberg, M. D., Benedek, M., Chen, Q., ... \& Silvia, P. J. (2018). Robust prediction of individual creative ability from brain functional connectivity. Proceedings of the National Academy of Sciences, 115, 1087-1092.

Beaty, R. E., Seli, P., \& Schacter, D. L. (2019). Network neuroscience of creative cognition: mapping cognitive mechanisms and individual differences in the creative brain. Current Opinion in Behavioral Sciences, 27, 22-30. 
Beaty, R. E., \& Silvia, P. J. (2012). Why do ideas get more creative across time? An executive interpretation of the serial order effect in divergent thinking tasks. Psychology of Aesthetics, Creativity, and the Arts, 6, 309-319.

Beaty, R. E., \& Silvia, P. J. (2013). Metaphorically speaking: Cognitive abilities and the production of figurative language. Memory \& Cognition, 41, 255-267.

Beaty, R. E., Silvia, P. J., Nusbaum, E. C., Jauk, E., \& Benedek, M. (2014b). The roles of associative and executive processes in creative cognition. Memory \& Cognition, 42, 1186-1197.

Beaty, R. E., Smeekens, B. A., Silvia, P. J., Hodges, D. A., \& Kane, M. J. (2013). A first look at the role of domain-general cognitive and creative abilities in jazz improvisation. Psychomusicology: Music, Mind, and Brain, 23, 262.

Benedek, M., Franz, F., Heene, M., \& Neubauer, A. C. (2012). Differential effects of cognitive inhibition and intelligence on creativity. Personality and Individual Differences, 53, 480-485.

Benedek M, \& Fink, A (2019). Toward a neurocognitive framework of creative cognition: The role of memory, attention, and cognitive control. Current Opinion in Behavioral Sciences, 27, 116-122.

Benedek, M., Jauk, E., Fink, A., Koschutnig, K., Reishofer, G., Ebner, F., \& Neubauer, A. C. (2014a). To create or to recall? Neural mechanisms underlying the generation of creative new ideas. NeuroImage, 88, 125-133.

Benedek, M., Jauk, E., Sommer, M., Arendasy, M., \& Neubauer, A. C. (2014b). Intelligence, creativity, and cognitive control: The common and differential involvement of executive functions in intelligence and creativity. Intelligence, 46, 73-83.

Benedek, M., Mühlmann, C., Jauk, E., \& Neubauer, A. C. (2013). Assessment of divergent thinking by means of the subjective top-scoring method: Effects of the number of top-ideas and time-on-task on reliability and validity. Psychology of Aesthetics, Creativity, and the Arts, 7, 341-349.

Benedek, M., \& Neubauer, A. C. (2013). Revisiting Mednick's model on creativity-related differences in associative hierarchies. Evidence for a common path to uncommon thought. The Journal of Creative Behavior, 47, 273-289. 
Benedek, M., Schües, T., Beaty, R. E., Jauk, E., Koschutnig, K., Fink, A., \& Neubauer, A. C. (2018). To create or to recall original ideas: Brain processes associated with the imagination of novel object uses. Cortex, 99, 93-102.

Berger, F. R., Gupta, W. B., Berger, R. M., \& Skinner, J. (1990). Air Force Officer Qualifying Test (AFOQT) form P manual. Tech. Rep. No. AFHRL-TR-89-56). Brooks Air Force Base, TX: Manpower and Personnel Division, Air Force Human Resources Laboratory.

Bingham, W. V. (1937). Aptitudes and aptitude testing. Oxford, England: Harpers.

Bowden, E. M., \& Beeman, M. J. (1998). Getting the right idea: Semantic activation in the right hemisphere may help solve insight problems. Psychological science, 9(6), 435-440.

Buckner, R. L., \& DiNicola, L. M. (2019). The brain's default network: Updated anatomy, physiology and evolving insights. Nature Reviews Neuroscience, 1-16.

Carroll, J. B. (1993). Human cognitive abilities: A survey of factor-analytic studies. New York: Cambridge University Press.

Cattell, R.B. \& Cattell, A.K.S. (1961/2008) Measuring intelligence with the Culture Fair Tests. Hogrefe, Oxford, UK.

Cattell, R. B. (1963). Theory of fluid and crystallized intelligence: A critical experiment. Journal of Educational Psychology, 54, 1-22.

Chen, Q., Beaty, R. E., Wei, D., Yang, J., Sun, J., Liu, W., ... \& Qiu, J. (2016). Longitudinal alterations of frontoparietal and frontotemporal networks predict future creative cognitive ability. Cerebral Cortex, 28, 103-115.

Christensen, A. P. (2018). NetworkToolbox: Methods and measures for brain, cognitive, and psychometric network analysis in R. The R Journal, 10, 422-439. https://doi.org/10.32614/RJ-2018-065

Christensen, A. P., \& Kenett, Y. N. (2019). Semantic network analysis (SemNA): A tutorial on preprocessing, estimating, and analyzing semantic networks. PsyArXiv.

Christensen, A. P., Silvia, P. J., Nusbaum, E. C., \& Beaty, R. E. (2018). Clever people: Intelligence and humor production ability. Psychology of Aesthetics, Creativity, and the Arts, 12, 136-143. 
Cho, Sun Hee, Jan Te Nijenhuis, Annelies EM van Vianen, HEUI-BAIK KIM, and Kun Ho Lee. "The relationship between diverse components of intelligence and creativity." The Journal of Creative Behavior 44, no. 2 (2010): 125-137.

Chrysikou, E. G. (2019). Creativity in and out of (cognitive) control. Current Opinion in Behavioral Sciences, 27, 94-99.

Cole, M. W., Ito, T., \& Braver, T. S. (2015). Lateral prefrontal cortex contributes to fluid intelligence through multinetwork connectivity. Brain Connectivity, 5, 497-504.

Colom, R., Juan-Espinosa, M., Abad, F., \& García, L. F. (2000). Negligible sex differences in general intelligence. Intelligence, 28, 57-68.

Conway, A. R., \& Kovacs, K. (2015). New and emerging models of human intelligence. Wiley Interdisciplinary Reviews: Cognitive Science, 6, 419-426.

Cox, R. W., \& Hyde, J. S. (1997). Software tools for analysis and visualization of fMRI data. NMR in Biomedicine: An International Journal Devoted to the Development and Application of Magnetic Resonance In Vivo, 10, 171-178.

Cropley, A. J. (2000). Defining and measuring creativity: Are creativity tests worth using?. Roeper Review, 23, 72-79.

Cropley, A. (2006). Functional creativity: A socially-useful creativity concept. Baltic Journal of Psychology, 7, 26-35.

Cumming, G. (2012). Understanding the new statistics: Effect sizes, confidence intervals, and metaanalysis. New York, NY: Routledge.

Dadi, K., Rahim, M., Abraham, A., Chyzhyk, D., Milham, M., Thirion, B., ... \& Alzheimer's Disease Neuroimaging Initiative. (2019). Benchmarking functional connectome-based predictive models for resting-state fMRI. NeuroImage, 192, 115-134.

Dale, A. M., Fischl, B., \& Sereno, M. I. (1999). Cortical surface-based analysis: I. Segmentation and surface reconstruction. Neuroimage, 9, 179-194. 
Deary, I. J., Penke, L., \& Johnson, W. (2010). The neuroscience of human intelligence differences. Nature reviews neuroscience, 11, 201-211.

Diedrich, J., Benedek, M., Jauk, E., \& Neubauer, A. C. (2015). Are creative ideas novel and useful?. Psychology of Aesthetics, Creativity, and the Arts, 9, 35-40.

Dygert, S. K., \& Jarosz, A. F. (2019). Individual differences in creative cognition. Journal of Experimental Psychology: General.

Ekstrom, R. B., Dermen, D., \& Harman, H. H. (1976). Manual for kit of factor-referenced cognitive tests (Vol. 102). Princeton, NJ: Educational testing service.

Esteban, O., Markiewicz, C. J., Blair, R. W., Moodie, C. A., Isik, A. I., Erramuzpe, A., ... \& Oya, H. (2019). fMRIPrep: A robust preprocessing pipeline for functional MRI. Nature Methods, 16, 111-116.

Finn, E. S., Shen, X., Scheinost, D., Rosenberg, M. D., Huang, J., Chun, M. M., ... \& Constable, R. T. (2015). Functional connectome fingerprinting: Identifying individuals using patterns of brain connectivity. Nature Neuroscience, 18, 1664-1671.

Fonov, V. S., Evans, A. C., McKinstry, R. C., Almli, C. R., \& Collins, D. L. (2009). Unbiased nonlinear average age-appropriate brain templates from birth to adulthood. NeuroImage, (47), S102.

Forthmann, B., Jendryczko, D., Scharfen, J., Kleinkorres, R., Benedek, M., \& Holling, H. (2019). Creative ideation, broad retrieval ability, and processing speed: A confirmatory study of nested cognitive abilities. Intelligence, 75, 59-72.

Goldstein, S., Princiotta, D., \& Naglieri, J. A. (2015). Handbook of intelligence. Evolutionary theory, historical perspective, and current concepts. New York, NY: Springer.

Gottfredson, L.S. (1997). Why g matters: The complexity of everyday life. Intelligence, 24, 79-132.

Gray, J. R., Chabris, C. F., \& Braver, T. S. (2003). Neural mechanisms of general fluid intelligence. Nature Neuroscience, 6(3), 316-322.

Green, A. E. (2016). Creativity, within reason: Semantic distance and dynamic state creativity in relational thinking and reasoning. Current Directions in Psychological Science, 25(1), 28-35. 
Greene, A. S., Gao, S., Scheinost, D., \& Constable, R. T. (2018). Task-induced brain state manipulation improves prediction of individual traits. Nature Communications, 9, 2807.

Greve, D. N., \& Fischl, B. (2009). Accurate and robust brain image alignment using boundary-based registration. NeuroImage, 48, 63-72.

Guilford, J. P. (1967). The nature of human intelligence. New York, NY, US: McGraw-Hill.

Haier, R.J., Siegel, B.V., Nuechterlein, K.H., Hazlett, E., Wu, J.C., Paek, J., Browning, H.L., Buchsbaum, M.S., 1988. Cortical glucose metabolic rate correlates of abstract reasoning and attention studied with positron emission tomography. Intelligence, 12, 199-217.

Halpern, D. F., \& LaMay, M. L. (2000). The smarter sex: A critical review of sex differences in intelligence. Educational Psychology Review, 12, 229-246.

Hsu, W. T., Rosenberg, M. D., Scheinost, D., Constable, R. T., \& Chun, M. M. (2018). Resting-state functional connectivity predicts neuroticism and extraversion in novel individuals. Social Cognitive and Affective Neuroscience, 13, 224-232.

Hunt, E. (2000). Let's hear it for crystallized intelligence. Learning and Individual Differences, 1, 123-129.

Jenkinson, M., Bannister, P., Brady, M., \& Smith, S. (2002). Improved optimization for the robust and accurate linear registration and motion correction of brain images. Neuroimage, 17, 825-841.

Jiang, R., Calhoun, V. D., Fan, L., Zuo, N., Jung, R., Qi, S., ... \& Fu, Z. (2020). Gender differences in connectome-based predictions of individualized intelligence quotient and sub-domain scores. Cerebral Cortex, 30, 888-900.

Jauk, E., Benedek, M., \& Neubauer, A. C. (2014). The road to creative achievement: A latent variable model of ability and personality predictors. European Journal of Personality, 28, 95-105.

Jauk, E., Benedek, M., Dunst, B., \& Neubauer, A. C. (2013). The relationship between intelligence and creativity: New support for the threshold hypothesis by means of empirical breakpoint detection. Intelligence, 41, 212-221.

Jensen, A. R. (1998). The g factor. Westport CT. Praeger. 
Jung, R. E. (2014). Evolution, creativity, intelligence, and madness: "Here Be Dragons". Frontiers in Psychology, 5, 784 .

Jung, R. E., \& Chohan, M. O. (2019). Three individual difference constructs, one converging concept: adaptive problem solving in the human brain. Current Opinion in Behavioral Sciences, 27, 163168.

Jung, R. E., Gasparovic, C., Chavez, R. S., Flores, R. A., Smith, S. M., Caprihan, A., \& Yeo, R. A. (2009). Biochemical support for the "threshold" theory of creativity: A magnetic resonance spectroscopy study. Journal of Neuroscience, 29, 5319-5325.

Jung, R. E., \& Haier, R. J. (2007). The Parieto-Frontal Integration Theory (P-FIT) of intelligence: Converging neuroimaging evidence. Behavioral and Brain Sciences, 30, 135-154.

Jung, R. E., Haier, R. J., Vartanian, O., Bristol, A. S., \& Kaufman, J. C. (2013). Creativity and intelligence: Brain networks that link and differentiate the expression of genius. Neuroscience of Creativity, 233-254.

Jung, R. E., Mead, B. S., Carrasco, J., \& Flores, R. A. (2013). The structure of creative cognition in the human brain. Frontiers in Human Neuroscience, 7, 330.

Jung, R. E., Segall, J. M., Bockholt, H. J., Flores, R. A., Smith, S. M., Chavez, R. S., \& Haier, R. J. (2010). Neuroanatomy of creativity. Human Brain Mapping, 31, 398-409.

Jung, R. E., \& Vartanian, O. (Eds.). (2018). The Cambridge handbook of the neuroscience of creativity. Cambridge University Press.

Kan, K. J., Kievit, R. A., Dolan, C., \& van der Maas, H. (2011). On the interpretation of the CHC factor Gc. Intelligence, 39, 292-302.

Karwowski, M., Dul, J., Gralewski, J., Jauk, E., Jankowska, D. M., Gajda, A., ... \& Benedek, M. (2016). Is creativity without intelligence possible? A necessary condition analysis. Intelligence, 57, 105-117.

Katz, L. N., Yates, J. L., Pillow, J. W., \& Huk, A. C. (2016). Dissociated functional significance of decisionrelated activity in the primate dorsal stream. Nature, 535, 285-288. 
Kell, H. J., Lubinski, D., Benbow, C. P., and Steiger, J. H. (2013). Creativity and technical innovation: Spatial ability's unique role. Psychological Science, 24, 1831-1836.

Kenett, Y. N., Medaglia, J. D., Beaty, R. E., Chen, Q., Betzel, R. F., Thompson-Schill, S. L., \& Qiu, J. (2018). Driving the brain towards creativity and intelligence: A network control theory analysis. Neuropsychologia, 118, 79-90.

Kenett, Y. N., \& Faust, M. (2019). A semantic network cartography of the creative mind. Trends in Cognitive Sciences, 23, 271-274.

Krakauer, J. W., Ghazanfar, A. A., Gomez-Marin, A., MacIver, M. A., \& Poeppel, D. (2017). Neuroscience needs behavior: correcting a reductionist bias. Neuron, 93, 480-490.

Kim, K. H. (2005) Can only intelligent people be creative? Journal of Secondary Gifted Education, 16, 5766.

Klein, A., Ghosh, S. S., Bao, F. S., Giard, J., Häme, Y., Stavsky, E., .. \& Keshavan, A. (2017). Mindboggling morphometry of human brains. PLoS Computational Biology, 13, e1005350.

Kleinmintz, O. M., Ivancovsky, T., \& Shamay-Tsoory, S. G. (2019). The twofold model of creativity: the neural underpinnings of the generation and evaluation of creative ideas. Current Opinion in Behavioral Sciences, 27, 131-138.

Kovacs, K., \& Conway, A. R. (2016). Process overlap theory: A unified account of the general factor of intelligence. Psychological Inquiry, 27, 151-177.

Lake, E. M., Finn, E. S., Noble, S. M., Vanderwal, T., Shen, X., Rosenberg, M. D., ... \& Constable, R. T. (2019). The functional brain organization of an individual allows prediction of measures of social abilities transdiagnostically in autism and attention-deficit/hyperactivity disorder. Biological Psychiatry, 86, 315-326.

Lee, K. H., Choi, Y. Y., Gray, J. R., Cho, S. H., Chae, J. H., Lee, S., \& Kim, K. (2006). Neural correlates of superior intelligence: Stronger recruitment of posterior parietal cortex. Neuroimage, 29, 578586. 
Lee, C. S., \& Therriault, D. J. (2013). The cognitive underpinnings of creative thought: A latent variable analysis exploring the roles of intelligence and working memory in three creative thinking processes. Intelligence, 41, 306-320.

Liu, S., Erkkinen, M. G., Healey, M. L., Xu, Y., Swett, K. E., Chow, H. M., \& Braun, A. R. (2015). Brain activity and connectivity during poetry composition: Toward a multidimensional model of the creative process. Human Brain Mapping, 36, 3351-3372.

Marder, E., \& Goaillard, J. M. (2006). Variability, compensation and homeostasis in neuron and network function. Nature Reviews Neuroscience, 7, 563-574.

McGrew, K. S. (2005). The Cattell-Horn-Carroll Theory of cognitive abilities: Past, present, and future. In D. P. Flanagan \& P. L. Harrison (Eds.), Contemporary Intellectual Assessment: Theories, Tests, and Issues (pp. 136-181). New York, NY, US: The Guilford Press.

McGrew, K. S. (2009). CHC theory and the human cognitive abilities project: Standing on the shoulders of the giants of psychometric intelligence research. Intelligence, 37, 1-10.

McNeish, D., \& Wolf, M. G. (2019). Sum scores are factor scores. PsyArXiv.

Mednick S. (1962). The associative basis of the creative process. Psychological Review, 69, 220-232.

Miller, D. I., \& Halpern, D. F. (2014). The new science of cognitive sex differences. Trends in Cognitive Sciences, $18,37-45$.

Neisser, U., Boodoo, G., Bouchard Jr, T. J., Boykin, A. W., Brody, N., Ceci, S. J., ... \& Urbina, S. (1996). Intelligence: knowns and unknowns. American Psychologist, 51, 77-101.

Neubauer, A. C., \& Fink, A. (2009). Intelligence and neural efficiency. Neuroscience \& Biobehavioral Reviews, 33, 1004-1023.

Niendam, T. A., Laird, A. R., Ray, K. L., Dean, Y. M., Glahn, D. C., \& Carter, C. S. (2012). Meta-analytic evidence for a superordinate cognitive control network subserving diverse executive functions. Cognitive, Affective, \& Behavioral Neuroscience, 12, 241-268.

Nusbaum, E. C., \& Silvia, P. J. (2011). Are intelligence and creativity really so different? Fluid intelligence, executive processes, and strategy use in divergent thinking. Intelligence, 39, 36-45. 
Pagnani, A. R. (2011). Gender differences. In Editors-in-Chief: M. A. Runco \& S. R. Pritzker (Eds.), Encyclopedia of creativity (second edition) (pp. 551-557). San Diego: Academic Press.

Pinho, A. L., de Manzano, Ö., Fransson, P., Eriksson, H., \& Ullén, F. (2014). Connecting to create: expertise in musical improvisation is associated with increased functional connectivity between premotor and prefrontal areas. Journal of Neuroscience, 34, 6156-6163.

Plucker, J. A., Esping, A., Kaufman, J. C., \& Avitia, M. J. (2015). Creativity and intelligence. In Handbook of intelligence (pp. 283-291). Springer, New York, NY.

Plucker, J.A., \& Renzulli, J. S. (1999). Psychometric approaches to the study of human creativity. Handbook of Creativity, 35, 61.

Power, J. D., Barnes, K. A., Snyder, A. Z., Schlaggar, B. L., \& Petersen, S. E. (2012). Spurious but systematic correlations in functional connectivity MRI networks arise from subject motion. NeuroImage, 59, 2142-2154.

Power, J. D., Mitra, A., Laumann, T. O., Snyder, A. Z., Schlaggar, B. L., \& Petersen, S. E. (2014). Methods to detect, characterize, and remove motion artifact in resting state fMRI. Neuroimage, 84, 320-341.

Rosenberg, M. D., Finn, E. S., Scheinost, D., Constable, R. T., \& Chun, M. M. (2017). Characterizing attention with predictive network models. Trends in Cognitive Sciences, 21, 290-302.

Rosenberg, M. D., Hsu, W. T., Scheinost, D., Todd Constable, R., \& Chun, M. M. (2018). Connectomebased models predict separable components of attention in novel individuals. Journal of Cognitive Neuroscience, 30, 160-173.

Rosseel, Y. (2012). Lavaan: An R package for structural equation modeling and more. Version 0.5-12 (BETA). Journal of Statistical Software, 48, 1-36.

R Core Team (2019). R: A language and environment for statistical computing. R Foundation for Statistical Computing, Vienna, Austria. Retrieved from https://www.R-project.org Runco M. A., \& Acar, S. (2012).

Divergent thinking as an indicator of creative potential. Creativity Research Journal, 24, 66-75. 
Runco, M. A., \& Acar, S. (2012). Divergent thinking as an indicator of creative potential. Creativity Research Journal, 24, 66-75.

Runco, M. A., \& Jaeger, G. J. (2012). The standard definition of creativity. Creativity Research Journal, 24, 92-96.

Ryman, S. G., van den Heuvel, M. P., Yeo, R. A., Caprihan, A., Carrasco, J., Vakhtin, A. A., ... \& Jung, R. E. (2014). Sex differences in the relationship between white matter connectivity and creativity. NeuroImage, 101, 380-389.

Satterthwaite, T. D., Elliott, M. A., Gerraty, R. T., Ruparel, K., Loughead, J., Calkins, M. E., ... \& Wolf, D. H. (2013). An improved framework for confound regression and filtering for control of motion artifact in the preprocessing of resting-state functional connectivity data. Neuroimage, 64, 240-256.

Schacter, D. L., Addis, D. R., \& Buckner, R. L. (2007). Remembering the past to imagine the future: the prospective brain. Nature Reviews Neuroscience, 8, 657-661.

Schaefer, A., Kong, R., Gordon, E. M., Laumann, T. O., Zuo, X. N., Holmes, A. J., ... \& Yeo, B. T. (2017). Local-global parcellation of the human cerebral cortex from intrinsic functional connectivity MRI. Cerebral Cortex, 28, 3095-3114.

Schmank, C. J., Goring, S. A., Kovacs, K., \& Conway, A. R. (2019). Psychometric Network Analysis of the Hungarian WAIS. Journal of Intelligence, 7, 21.

Schneider, W. J., \& McGrew, K. S. (2012). The Cattell-Horn-Carroll model of intelligence. In D. P. Flanagan, \& P. L. Harrison (Eds.), Contemporary intellectual assessment: Theories, tests, and issues (3rd ed., pp. 99-144). New York, NY: Guilford Press.

Schultz, D. H., \& Cole, M. W. (2016). Higher intelligence is associated with less task-related brain network reconfiguration. Journal of Neuroscience, 36, 8551-8561.

Shen, X., Finn, E. S., Scheinost, D., Rosenberg, M. D., Chun, M. M., Papademetris, X., \& Constable, R. T. (2017). Using connectome-based predictive modeling to predict individual behavior from brain connectivity. Nature Protocols, 12, 506. 
Shipstead, Z., Harrison, T. L., \& Engle, R. W. (2016). Working memory capacity and fluid intelligence: Maintenance and disengagement. Perspectives on Psychological Science, 11, 771-799.

Shouksmith, G. (1973). Intelligence, creativity and cognitive style. London: Angus \& Robertson.

Silvia, P. J. (2008). Another look at creativity and intelligence: exploring higher-order models and probable confounds. Personality and Individual Differences, 44, 1012-1021.

Silvia, P. J. (2015). Intelligence and creativity are pretty similar after all. Educational Psychology Review, 27, 599-606.

Silvia, P. J., \& Beaty, R. E. (2012). Making creative metaphors: The importance of fluid intelligence for creative thought. Intelligence, 40, 343-351.

Silvia, P. J., Beaty, R. E., \& Nusbaum, E. C. (2013). Verbal fluency and creativity: General and specific contributions of broad retrieval ability (Gr) factors to divergent thinking. Intelligence, 41, 328-340.

Silvia, P. J., Beaty, R. E., Nusbaum, E. C., Eddington, K. M., Levin-Aspenson, H., \& Kwapil, T. R. (2014). Everyday creativity in daily life: An experience-sampling study of "little c" creativity. Psychology of Aesthetics, Creativity, and the Arts, 8, 183-188.

Silvia, P. J., Martin, C., \& Nusbaum, E. C. (2009). A snapshot of creativity: Evaluating a quick and simple method for assessing divergent thinking. Thinking Skills and Creativity, 4, 79-85.

Silvia, P. J., Nusbaum, E. C., \& Beaty, R. E. (2017). Old or new? Evaluating the old/new scoring method for divergent thinking tasks. The Journal of Creative Behavior, 51, 216-224.

Silvia, P. J., Winterstein, B. P., Willse, J. T., Barona, C. M., Cram, J. T., Hess, K. I., ... \& Richard, C. A. (2008). Assessing creativity with divergent thinking tasks: Exploring the reliability and validity of new subjective scoring methods. Psychology of Aesthetics, Creativity, and the Arts, 2, 68-85.

Spearman, C. (1904). "General intelligence," objectively determined and measured. American Journal of Psychology, 15, 201-292.

Sripada, C., Angstadt, M., Rutherford, S., Kessler, D., Kim, Y., Yee, M., \& Levina, E. (2019). Basic Units of Inter-Individual Variation in Resting State Connectomes. Scientific Reports, 9, 1900. 
Sternberg, R. J. (1984). Toward a triarchic theory of human intelligence. Behavioral and Brain Sciences, 7, 269-287.

Sternberg, R. J. (1999). The theory of successful intelligence. Review of General Psychology, 3, 292-316.

Sternberg, R. J. (2008). Increasing fluid intelligence is possible after all. Proceedings of the National Academy of Sciences, 105, 6791-6792.

Sternberg, R. J., \& Kaufman, S. B. (Eds.). (2011). The Cambridge handbook of intelligence. Cambridge University Press.

Sternberg, R. J., \& Lubart, T. I. (1991). An investment theory of creativity and its development. Human Development, 34, 1-31.

Sternberg, R. J., \& O’Hara, L. A. (1999). Creativity and intelligence. In R. J. Sternberg (Ed.), Handbook of creativity (pp. 251-272). Cambridge, MA: Cambridge University Press.

Takeuchi, H., Taki, Y., Matsudaira, I., Ikeda, S., Kawata, K. H. D. S., Nouchi, R., ... \& Araki, T. (2020). Convergent creative thinking performance is associated with white matter structures: Evidence from a large sample study. NeuroImage, 210, 116577.

Takeuchi, H., Taki, Y., Nouchi, R., Yokoyama, R., Kotozaki, Y., Nakagawa, S., ... \& Araki, T. (2017). Regional homogeneity, resting-state functional connectivity and amplitude of low frequency fluctuation associated with creativity measured by divergent thinking in a sex-specific manner. NeuroImage, 152, 258-269.

Takeuchi, H., Taki, Y., Sassa, Y., Hashizume, H., Sekiguchi, A., Fukushima, A., \& Kawashima, R. (2010). White matter structures associated with creativity: evidence from diffusion tensor imaging. Neuroimage, 51, 11-18.

Terman, L. M. (1922). A new approach to the study of genius. Psychological Review, 29, 310.

Thurstone, L. L. (1938). Primary mental abilities. Psychometric Monographs, 1, ix + 121.

Tulving, E. (1972). Episodic and semantic memory. In E. Tulving \& W. Donaldson (Eds.), Organization of Memory (pp. 381-403). New York: Academic Press. 
Uddin, L. Q. (2015). Salience processing and insular cortical function and dysfunction. Nature Reviews Neuroscience, 16, 55-61.

Van Den Heuvel, M. P., Stam, C. J., Kahn, R. S., \& Pol, H. E. H. (2009). Efficiency of functional brain networks and intellectual performance. Journal of Neuroscience, 29, 7619-7624.

Van Der Maas, H. L., Dolan, C. V., Grasman, R. P., Wicherts, J. M., Huizenga, H. M., \& Raijmakers, M. E. (2006). A dynamical model of general intelligence: The positive manifold of intelligence by mutualism. Psychological Review, 113, 842.

Vartanian, O., Beatty, E. L., Smith, I., Blackler, K., Lam, Q., \& Forbes, S. (2018). One-way traffic: The inferior frontal gyrus controls brain activation in the middle temporal gyrus and inferior parietal lobule during divergent thinking. Neuropsychologia, 118, 68-78.

Volle, E. (2018). Associative and controlled cognition in divergent thinking: theoretical, experimental, neuroimaging evidence, and new directions. In R. E. Jung \& O. Vartanian (eds.), The Cambridge handbook of the neuroscience of creativity, 333-362. Cambridge: Cambridge University Press.

Wai, J., Lubinski, D., and Benbow, C. P. (2009). Spatial ability for STEM domains: Aligning over fifty years of cumulative psychological knowledge solidifies its importance. Journal of Education Psychology, 101, 817-835.

Weiler, J. A., Suchan, B., \& Daum, I. (2010). When the future becomes the past: Differences in brain activation patterns for episodic memory and episodic future thinking. Behavioural Brain Research, 212, 196-203.

Whitfield-Gabrieli, S., \& Nieto-Castanon, A. (2012). Conn: A functional connectivity toolbox for correlated and anticorrelated brain networks. Brain Connectivity, 2, 125-141.

Yves Rosseel (2012). lavaan: An R Package for Structural Equation Modeling. Journal of Statistical Software, 48(2), 1-36. URL http://www.jstatsoft.org/v48/i02/.

Wu, E. X., Liaw, G. J., Goh, R. Z., Chia, T. T., Chee, A. M., Obana, T., ... \& Asplund, C. L. (2020). Overlapping attentional networks yield divergent behavioral predictions across tasks: Neuromarkers for diffuse and focused attention?. NeuroImage, 116535. 
Zeng, L., Proctor, R. W., \& Salvendy, G. (2011). Can traditional divergent thinking tests be trusted in measuring and predicting real-world creativity?. Creativity Research Journal, 23, 24-3.

Zhang, Y., M. Brady, and S. Smith. (2001). Segmentation of Brain MR Images Through a Hidden Markov Random Field Model and the Expectation-Maximization Algorithm. IEEE Transactions on Medical Imaging, 20, 45-57. 
Table 1

Descriptive statistics for the intelligence tasks

\begin{tabular}{|c|c|c|c|c|c|}
\hline Task & $M$ & $S D$ & $\begin{array}{l}R(\min - \\
\max )\end{array}$ & Skew & Kurtosis \\
\hline $\begin{array}{l}\text { Gc_advanced } \\
\text { vocabulary }\end{array}$ & 9.02 & 2.86 & $1-17$ & -0.18 & 0.19 \\
\hline $\begin{array}{l}\text { Gc_extended range } \\
\text { vocabulary }\end{array}$ & 12.12 & 3.37 & $3-20$ & -0.06 & -0.20 \\
\hline $\begin{array}{l}\text { Gf_series } \\
\text { completion }\end{array}$ & 7.97 & 1.63 & $3-11$ & -0.57 & 0.31 \\
\hline Gf_letter sets & 8.93 & 2.21 & $1-14$ & -0.29 & 0.44 \\
\hline Gf_number series & 9.55 & 2.69 & $3-15$ & -0.05 & -0.66 \\
\hline Gr_animals & 19.40 & 4.28 & $6-34$ & 0.01 & 0.15 \\
\hline Gr_good & 7.45 & 2.69 & $1-16$ & 0.56 & 0.53 \\
\hline Gr_hot & 5.82 & 2.34 & $2-14$ & 0.80 & 0.67 \\
\hline Gr_occupation & 14.35 & 3.36 & $3-23$ & -0.23 & 0.40 \\
\hline Gr_vegetable & 15.67 & 3.66 & $9-25$ & 0.34 & 0.50 \\
\hline Gv_block rotations & 3.81 & 2.17 & $0-10$ & 0.49 & -0.39 \\
\hline $\begin{array}{l}\text { Gv_cube } \\
\text { comparisons }\end{array}$ & 13.15 & 3.70 & $3-20$ & -0.47 & -0.32 \\
\hline Gv_paper folding & 5.30 & 2.19 & $1-10$ & -0.05 & -0.75 \\
\hline
\end{tabular}

Notes. $\mathrm{Gc}=$ crystallized intelligence; $\mathrm{Gf}=$ fluid intelligence; $\mathrm{Gr}=$ broad retrieval ability; $\mathrm{Gv}=$ visuospatial intelligence. 
Table 2

Descriptive statistics for the divergent thinking tasks

\begin{tabular}{lccccc}
\hline \multicolumn{1}{c}{ Task } & $M$ & $S D$ & $\begin{array}{c}R(\min - \\
\max )\end{array}$ & Skew & Kurtosis \\
\hline DT_mri_r1 & 2.29 & 0.39 & $1.22-3.78$ & 0.44 & 0.79 \\
DT_mri_r2 & 1.46 & 0.24 & $1-2.37$ & 1.04 & 1.40 \\
DT_mri_r3 & 1.91 & 0.29 & $1-2.70$ & 0.03 & -0.16 \\
DT_mri_r4 & 1.76 & 0.38 & $1-3.36$ & 1.08 & 2.17 \\
\hline DT1_r1 & 1.90 & 0.47 & $1-3.25$ & 0.59 & 0.23 \\
DT1_r2 & 1.45 & 0.33 & $1-2.67$ & 0.95 & 0.55 \\
DT1_r3 & 1.59 & 0.37 & $1-3.33$ & 1.19 & 3.12 \\
DT1_r4 & 1.39 & 0.42 & $1-3$ & 1.43 & 2.02 \\
\hline DT2_r1 & 1.75 & 0.54 & $1-3.67$ & 1.10 & 1.03 \\
DT2_r2 & 1.50 & 0.41 & $1-3.5$ & 1.77 & 4.82 \\
DT2_r3 & 1.69 & 0.44 & $1-3.5$ & 1.05 & 1.48 \\
DT2_r4 & 1.34 & 0.43 & $1-4$ & 2.55 & 9.44 \\
\hline
\end{tabular}

Notes. $\mathrm{dt} 1=$ divergent thinking, box; $\mathrm{dt} 2=$ divergent thinking, rope; $\mathrm{dt} \_$mri $=$divergent thinking, MRI; r1-r4 = rater 1-rater 4. 
Table 3

Correlations between latent intelligence facets and divergent thinking

\begin{tabular}{|c|c|c|c|c|c|}
\hline & DT & Gc & Gf & Gr & Gv \\
\hline DT & 1 & & & & \\
\hline Gc & $.54[.37, .71]$ & 1 & & & \\
\hline Gf & $.39[.20, .57]$ & $.42[.22, .63]$ & 1 & & \\
\hline Gr & $.32[.14, .50]$ & $.51[.33, .68]$ & $.48[.30, .65]$ & 1 & \\
\hline Gv & $.41[.24, .59]$ & $.40[.21, .59]$ & $.59[.42, .76]$ & $.24[.04, .43]$ & 1 \\
\hline
\end{tabular}

Notes. DT = divergent thinking creativity; Gc = crystallized intelligence; $\mathrm{Gf}=$ fluid intelligence; $\mathrm{Gr}=$ broad retrieval ability; $\mathrm{Gv}=$ visuospatial intelligence. $\mathrm{N}=186$. 
Table 4

Top 10 high-degree nodes within individual divergent thinking and intelligence CPM networks

\begin{tabular}{|c|c|c|c|c|c|}
\hline \multicolumn{6}{|c|}{ DT Network } \\
\hline \# & $K$ & Network & $\mathbf{L} / \mathbf{R}$ & Lobe & Schaefer Atlas Node \# \\
\hline 1 & 52 & Control & $\mathrm{L}$ & Prefrontal & 105 \\
\hline 2 & 35 & Visual & $\mathrm{L}$ & Occipital & 15 \\
\hline 3 & 34 & Visual & $\mathrm{R}$ & Occipital & 164 \\
\hline 4 & 30 & Ventral Attention & $\mathrm{R}$ & Prefrontal & 228 \\
\hline 5 & 28 & Default & $\mathrm{L}$ & Prefrontal & 133 \\
\hline 6 & 24 & Control & $\mathrm{R}$ & Prefrontal & 256 \\
\hline 7 & 24 & Somatomotor & $\mathrm{R}$ & Frontal & 193 \\
\hline 8 & 24 & Control & $\mathrm{L}$ & Prefrontal & 107 \\
\hline 9 & 23 & Control & $\mathrm{R}$ & Prefrontal & 259 \\
\hline 10 & 21 & Ventral Attention & $\mathrm{L}$ & Frontal & 79 \\
\hline \multicolumn{6}{|c|}{ Gc Network } \\
\hline 1 & 37 & Visual & $\mathrm{L}$ & Occipital & 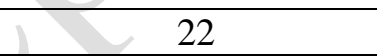 \\
\hline 2 & 26 & Visual & $\mathrm{L}$ & Occipital & 2 \\
\hline 3 & 21 & Control & $\mathrm{R}$ & Prefrontal & 256 \\
\hline 4 & 18 & Visual & $\mathrm{R}$ & Occipital & 172 \\
\hline 5 & 15 & Ventral Attention & $\mathrm{R}$ & Parietal & 222 \\
\hline 6 & 14 & Ventral Attention & $\mathrm{L}$ & Frontal & 79 \\
\hline 7 & 14 & Somatomotor & $\mathrm{L}$ & Temporal & 25 \\
\hline 8 & 12 & Visual & $\mathrm{R}$ & Occipital & 171 \\
\hline 9 & 12 & Default & $\mathrm{L}$ & Temporal & 116 \\
\hline 10 & 12 & Somatomotor & $\mathrm{L}$ & Frontal & 44 \\
\hline \multicolumn{6}{|c|}{ Gf Network } \\
\hline 1 & 39 & Visual & $\mathrm{R}$ & Occipital & 164 \\
\hline 2 & 37 & Visual & $\mathrm{L}$ & Occipital & 15 \\
\hline 3 & 33 & Visual & $\mathrm{L}$ & Occipital & 160 \\
\hline 4 & 31 & Visual & $\mathrm{R}$ & Occipital & 11 \\
\hline 5 & 24 & Default & $\mathrm{L}$ & Temporal & 119 \\
\hline 6 & 23 & Dorsal Attention & $\mathrm{R}$ & Parietal & 208 \\
\hline 7 & 22 & Visual & $\mathrm{R}$ & Occipital & 172 \\
\hline 8 & 20 & Control & $\mathrm{R}$ & Prefrontal & 256 \\
\hline 9 & 17 & Control & $\mathrm{R}$ & Prefrontal & 259 \\
\hline 10 & 17 & Ventral Attention & $\mathrm{L}$ & Prefrontal & 78 \\
\hline 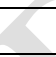 & \multicolumn{5}{|c|}{ Gr Network } \\
\hline 1 & 23 & Control & $\mathrm{L}$ & Limbic & 111 \\
\hline 2 & 17 & Ventral Attention & $\mathrm{R}$ & Limbic & 233 \\
\hline 4 & 16 & Default & $\mathrm{L}$ & Prefrontal & 138 \\
\hline 3 & 15 & Somatomotor & $\mathrm{R}$ & Frontal & 193 \\
\hline 5 & 11 & Control & $\mathrm{R}$ & Parietal & 266 \\
\hline 6 & 11 & Default & $\mathrm{L}$ & Prefrontal & 128 \\
\hline 7 & 8 & Dorsal Attention & $\mathrm{L}$ & Temporal & 56 \\
\hline 8 & 7 & Visual & $\mathrm{R}$ & Occipital & 154 \\
\hline 9 & 7 & Visual & $\mathrm{R}$ & Occipital & 153 \\
\hline 10 & 7 & Limbic & $\mathrm{L}$ & Temporal & 93 \\
\hline
\end{tabular}




\begin{tabular}{|c|c|c|c|c|c|}
\hline \multicolumn{6}{|c|}{ Gv Network } \\
\hline 1 & 25 & Visual & $\mathrm{R}$ & Occipital & 164 \\
\hline 2 & 22 & Control & $\mathrm{L}$ & Limbic & 110 \\
\hline 3 & 21 & Default & $\mathrm{L}$ & Parietal & 147 \\
\hline 4 & 20 & Control & $\mathrm{R}$ & Prefrontal & 259 \\
\hline 5 & 16 & Default & $\mathrm{R}$ & Prefrontal & 292 \\
\hline 6 & 16 & Visual & $\mathrm{R}$ & Visual & 172 \\
\hline 7 & 16 & Default & $\mathrm{L}$ & Limbic & 149 \\
\hline 8 & 14 & Default & $\mathrm{R}$ & Prefrontal & 284 \\
\hline 9 & 14 & Control & $\mathrm{R}$ & Prefrontal & 270 \\
\hline 10 & 14 & Somatomotor & $\mathrm{R}$ & Parietal & 197 \\
\hline \multicolumn{6}{|c|}{ G Network } \\
\hline 1 & 40 & Visual & $\mathrm{L}$ & Occipital & 15 \\
\hline 2 & 36 & Visual & $\mathrm{L}$ & Occipital & 22 \\
\hline 3 & 32 & Visual & $\mathrm{R}$ & Occipital & 164 \\
\hline 4 & 30 & Control & $\mathrm{R}$ & Prefrontal & 256 \\
\hline 5 & 24 & Visual & $\mathrm{R}$ & Occipital & 172 \\
\hline 6 & 24 & Ventral Attention & $\mathrm{L}$ & Frontal & 79 \\
\hline 7 & 23 & Dorsal Attention & $\mathrm{R}$ & Parietal & 205 \\
\hline 8 & 22 & Ventral Attention & $\mathrm{L}$ & Prefrontal & 78 \\
\hline 9 & 21 & Control & $\mathrm{L}$ & Limbic & 110 \\
\hline 10 & 20 & Ventral Attention & $\mathrm{R}$ & Limbic & 233 \\
\hline
\end{tabular}

Notes. The Schaefer atlas (300 nodes, 7 networks) was used to construct functional networks (Schaefer et al., 2018). $\mathrm{DT}=$ divergent thinking creativity; $\mathrm{Gc}=$ crystallized intelligence; $\mathrm{Gf}=$ fluid intelligence; $\mathrm{Gr}=$ broad retrieval ability; $\mathrm{Gv}=$ visuospatial intelligence. $\mathrm{K}=$ degree; $\mathrm{L}=$ left; $\mathrm{R}=$ right. 
Table 5

Top-10 high-degree nodes within combined divergent thinking and intelligence CPM networks

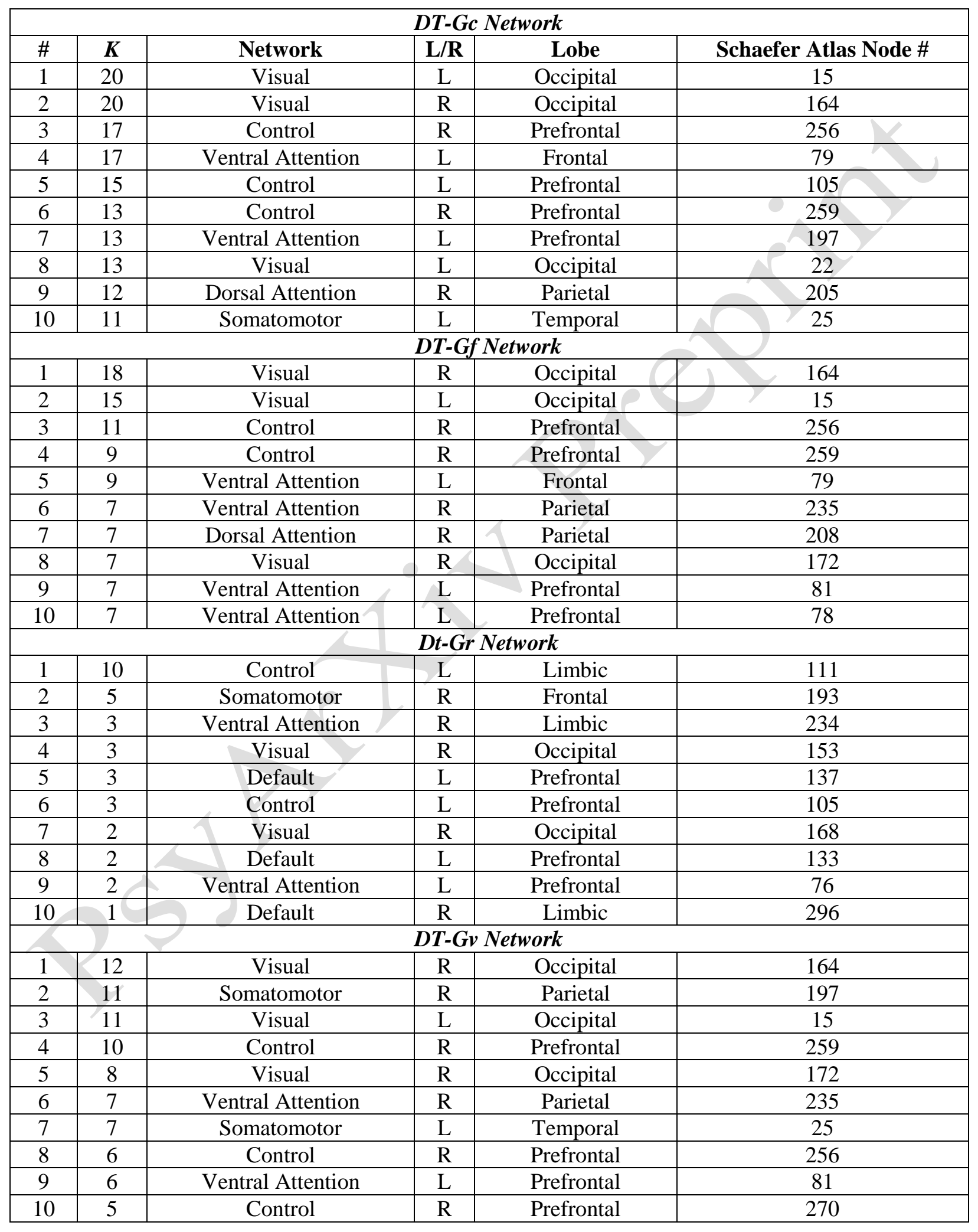




\begin{tabular}{|c|c|c|c|c|c|}
\hline \multicolumn{7}{|c|}{ DT-G Network } \\
\hline 1 & 1 & Visual & R & Occipital & 164 \\
\hline 2 & 2 & Visual & L & Occipital & 15 \\
\hline 3 & 3 & Control & R & Prefrontal & 256 \\
\hline 4 & 4 & Ventral Attention & L & Parietal & 105 \\
\hline 5 & 5 & Control & L & Prefrontal & 259 \\
\hline 6 & 6 & Control & R & Prefrontal & 197 \\
\hline 7 & 7 & Somatomotor & R & Parietal & 22 \\
\hline 8 & 8 & Visual & L & Occipital & 193 \\
\hline 9 & 9 & Dorsal Attention & R & Parietal & Frontal \\
\hline 10 & 10 & Somatomotor & R & F \\
\hline
\end{tabular}

Notes. The Schaefer atlas (300 nodes, 7 networks) was used to construct functional networks (Schaefer et al., 2018). DT = divergent thinking creativity; $\mathrm{Gc}=$ crystallized intelligence; $\mathrm{Gf}=$ fluid intelligence; $\mathrm{Gr}=$ broad retrieval ability; $\mathrm{Gv}=$ visuospatial intelligence. $\mathrm{K}=$ degree; $\mathrm{L}=$ left; $\mathrm{R}=$ right. 
Supplemental Table 1

\begin{tabular}{|c|c|c|c|c|c|c|c|c|c|c|c|c|c|c|c|c|c|c|c|c|c|c|c|c|c|}
\hline & 1 & 2 & 3 & 4 & 5 & 6 & 7 & 8 & 9 & 10 & 11 & 12 & 13 & 14 & 15 & 16 & 17 & 18 & 19 & 20 & 21 & 22 & 23 & 24 & 25 \\
\hline 1. dt_mri_r1 & 1 & & & & & & & & & & & & & & & & & & & 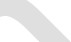 & U & & & & \\
\hline 2. dt_mri_r2 & 0.82 & 1 & & & & & & & & & & & & & & & & 0 & h & & 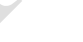 & & & & \\
\hline 3. dt_mri_r3 & 0.87 & 0.77 & 1 & & & & & & & & & & & & & & & & & $y$ & & & & & \\
\hline 4. dt_mri_r4 & 0.84 & 0.81 & 0.79 & 1 & & & & & & & & & & & & & & r & & & & & & & \\
\hline 5. dt1_r1 & 0.51 & 0.44 & 0.40 & 0.44 & 1 & & & & & & & & & & & & & 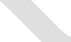 & & & & & & & \\
\hline 6. dt1_r2 & 0.34 & 0.25 & 0.25 & 0.33 & 0.72 & 1 & & & & & & & & & & & & 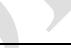 & & & & & & & \\
\hline 7. dt1_r3 & 0.46 & 0.41 & 0.39 & 0.40 & 0.67 & 0.57 & 1 & & & & & & & & & & 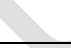 & 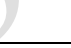 & & & & & & & \\
\hline 8. dt1_r4 & 0.41 & 0.36 & 0.34 & 0.37 & 0.77 & 0.70 & 0.66 & 1 & & & & & & & & 2 & & & & & & & & & \\
\hline 9. dt2_r1 & 0.43 & 0.31 & 0.41 & 0.45 & 0.45 & 0.38 & 0.43 & 0.41 & 1 & & & & & & $P$ & & & & & & & & & & \\
\hline 10. dt2_r2 & 0.34 & 0.20 & 0.32 & 0.32 & 0.42 & 0.38 & 0.44 & 0.47 & 0.78 & 1 & & & & . & 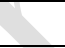 & & & & & & & & & & \\
\hline 11. dt2_r3 & 0.46 & 0.32 & 0.43 & 0.46 & 0.48 & 0.44 & 0.55 & 0.49 & 0.87 & 0.79 & 1 & & 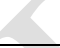 & 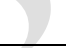 & & 1 & & & & & & & & & \\
\hline 12. dt2_r4 & 0.28 & 0.20 & 0.27 & 0.33 & 0.35 & 0.39 & 0.49 & 0.47 & 0.73 & 0.67 & 0.76 & 1 & 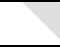 & + & & & & & & & & & & & \\
\hline 13. gc_adv & 0.25 & 0.20 & 0.20 & 0.27 & 0.30 & 0.32 & 0.28 & 0.25 & 0.27 & 0.20 & 0.18 & 0.21 & 1 & 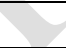 & & & & & & & & & & & \\
\hline 14. gc_ex & 0.31 & 0.27 & 0.27 & 0.27 & 0.25 & 0.25 & 0.24 & 0.18 & 0.25 & 0.21 & 0.22 & 0.16 & 0.49 & 1 & & & & & & & & & & & \\
\hline 15. gf_cfiq & 0.07 & 0.10 & 0.21 & 0.07 & 0.17 & 0.17 & 0.14 & 0.08 & 0.11 & 0.04 & 0.08 & 0.08 & 0.09 & 0.10 & 1 & & & & & & & & & & \\
\hline 16. gf_letters & 0.14 & 0.15 & 0.21 & 0.11 & 0.24 & 0.18 & 0.15 & 0.16 & 0.17 & 0.06 & 0.08 & 0.08 & 0.07 & 0.20 & 0.27 & 1 & & & & & & & & & \\
\hline 17. gf_numbers & 0.20 & 0.20 & 0.14 & 0.20 & 0.19 & 0.17 & 0.24 & 0.12 & 0.20 & 0.16 & 0.20 & 0.17 & 0.14 & 0.21 & 0.30 & 0.38 & 1 & & & & & & & & \\
\hline 18. gr_animals & 0.14 & 0.12 & 0.21 & 0.19 & 0.13 & 0.07 & 0.16 & 0.10 & 0.11 & 0.07 & 0.12 & 0.07 & 0.18 & 0.07 & 0.08 & 0.09 & 0.17 & 1 & & & & & & & \\
\hline 19. gr_good & 0.14 & 0.13 & 0.19 & 0.14 & 0.10 & 0.05 & 0.13 & 0.04 & 0.05 & 0.07 & 0.07 & -0.07 & 0.09 & 0.18 & 0.06 & 0.08 & -0.08 & 0.26 & 1 & & & & & & \\
\hline 20. gr_hot & 0.07 & 0.13 & 0.19 & 0.17 & 0.10 & 0.17 & 0.13 & 0.12 & 0.23 & 0.15 & 0.24 & 0.14 & 0.06 & 0.22 & 0.16 & 0.12 & 0.13 & 0.23 & 0.33 & 1 & & & & & \\
\hline 21. gr_jobs & 0.25 & 0.22 & 0.23 & 0.26 & 0.17 & 0.19 & 0.22 & 0.15 & 0.14 & 0.14 & 0.20 & 0.16 & 0.16 & 0.16 & 0.03 & 0.09 & 0.26 & 0.46 & 0.23 & 0.19 & 1 & & & & \\
\hline 22. gr_veg & 0.22 & 0.25 & 0.28 & 0.23 & 0.20 & 0.15 & 0.19 & 0.12 & 0.25 & 0.18 & 0.29 & 0.14 & 0.25 & 0.32 & 0.13 & 0.16 & 0.16 & 0.49 & 0.35 & 0.31 & 0.46 & 1 & & & \\
\hline 23. gv_blocks & 0.18 & 0.26 & 0.18 & 0.28 & 0.23 & 0.16 & 0.14 & 0.12 & 0.34 & 0.23 & 0.26 & 0.18 & 0.21 & 0.33 & 0.26 & 0.33 & 0.32 & 0.12 & 0.00 & 0.00 & 0.02 & 0.10 & 1 & & \\
\hline 24. gv_cubes & 0.21 & 0.21 & 0.18 & 0.17 & 0.20 & 0.12 & 0.14 & 0.15 & 0.14 & 0.16 & 0.17 & 0.16 & 0.05 & 0.16 & 0.06 & 0.30 & 0.27 & 0.15 & 0.08 & 0.02 & 0.10 & 0.20 & 0.33 & 1 & \\
\hline 25. gv_paper & 0.20 & 0.22 & 0.23 & 0.24 & 0.30 & 0.17 & 0.15 & 0.17 & 0.21 & 0.19 & 0.19 & 0.13 & 0.13 & 0.30 & 0.15 & 0.10 & 0.09 & 0.10 & 0.09 & 0.04 & -0.04 & 0.21 & 0.55 & 0.29 & 1 \\
\hline
\end{tabular}

Notes. Pearson correlations beween all observed variables. $\mathrm{dt} 1=$ divergent thinking, box; dt2 = divergent thinking, rope; $\mathrm{dt} \_$mri = divergent thinking, MRI; 1 $=$ rater $1 ; \mathrm{gc} \_a d v=$ crystallized intelligence, advanced vocabulary; gc_ext = crystallized intelligence, extended range; gf_cfiq = fluid intelligence, Cattell Series Completion; gf_lets = fluid intelligence, letter sets; gf_nums = fluid intelligence, number series; gr_ani = broad retrieval ability, animal category fluency; gr_good

= broad retrieval ability, synonyms for 'good'; gr_jobs = broad retrieval ability, occupations category; gr_hot = broad retrieval ability, synonyms for 'hot; gr_veg = broad retrieval ability, fruits and vegetables category; gv_block = visuospatial intelligence, block rotation; gv_cubes = visuospatial intelligence, cube comparison; gv_paper = visuospatial intelligence, paper folding. 